\title{
DIRAC COHOMOLOGY FOR DEGENERATE AFFINE HECKE-CLIFFORD ALGEBRAS
}

\author{
KEI YUEN CHAN
}

\author{
Korteweg-de Vries \\ Institute for Mathematics \\ Universiteit van Amsterdam \\ Science Park 105-107, 1098 XG \\ Amsterdam, The Netherlands \\ K.Y.Chan@uva.nl
}

\begin{abstract}
In this paper, we study the Dirac cohomology theory on a class of algebraic structures. The main examples of this algebraic structure are the degenerate affine HeckeClifford algebra of type $A_{n-1}$ by Nazarov and of classical types by Khongsap-Wang. The algebraic structure contains a remarkable subalgebra, which usually refers to Sergeev algebra for type $A_{n-1}$.

We define an analogue of the Dirac operator for those algebraic structures. A main result is to relate the central characters of modules of those algebras with the central characters of modules of the Sergeev algebra via the Dirac cohomology. The action of the Dirac operator on certain modules is also computed. Results in this paper could be viewed as a projective version of the Dirac cohomology of the degenerate affine Hecke algebra.
\end{abstract}

\section{Introduction}

Throughout this paper, we work over the ground field $\mathbb{C}$. Let $W$ be a Weyl group. It is well known that $W$ admits a non-trivial central extension

$$
1 \rightarrow \mathbb{Z}_{2} \rightarrow \widetilde{W} \rightarrow W \rightarrow 1
$$

where $\widetilde{W}$ is a distinguished double cover of $W$. The projective representations of $W$ are linear representations of $\widetilde{W}$ which do not factor through $W$. Those representations over $\mathbb{C}$ have been has been known for a long time from the work of Schur, Morris, Read, Stembridge, and others [Mo1], [Mo2], [Re], [Sc], [St].

The degenerate affine Hecke-Clifford algebra for type $A_{n-1}$ (see Definition 4.2) was introduced by Nazarov [Na] to study Young's symmetrizers of the projective representations of $S_{n}$. The degenerate affine Hecke-Clifford algebra for other classical types was later constructed by Khongsap-Wang [WK]. Those algebras could

DOI: $10.1007 / \mathrm{s} 00031-016-9390-9$

Received March 2, 2015. Accepted April 20, 2015.

Published online May 17, 2016.

Corresponding Author: K. Y. Chan, e-mail: K.Y.Chan@uva.nl. 
be viewed as the projective counterpart of the degenerate affine Hecke algebra of Lusztig.

The purpose of this paper is to establish Dirac cohomology theory for those classes of algebras. We first single out the algebraic structure (see Section 3 ) that is necessary to prove several important results for the Dirac cohomology, and then we show that the degenerate affine Hecke-Clifford algebras considered in $[\mathrm{Na}]$ and [WK] satisfy that algebraic structure. Our approach is an analogue of the one recently developed for degenerate affine Hecke algebras by Barbasch-CiubotaruTrapa $[\mathrm{BCT}]$ (also see a recent extension by Ciubotaru [Ci2]).

In more detail, let $\mathcal{H}_{W}$ be the associative algebra with certain important properties (see Definitions 3.1 and 3.3). The algebra $\mathcal{H}_{W}$ contains a remarkable subalgebra, namely $\operatorname{Seg}(W)$ (see again Definition 3.1), which is is the same as the Sergeev algebra when $W$ is of type $\mathrm{A}_{n-1}$.) The Dirac type element in $\mathcal{H}_{W}$ is defined as an analogue of the one in $[\mathrm{BCT}]$ and has some nice properties. In specific examples of $\mathcal{H}_{W}$ in Section 4 , the Dirac type element can be viewed as the square root of a certain Casmir type element (Theorem 4.23).

For an $\mathcal{H}_{W}$-module $(\pi, X)$, the Dirac cohomology is defined as

$$
H_{D}(X)=\operatorname{ker} \pi(D) /(\operatorname{ker} \pi(D) \cap \operatorname{im} \pi(D)),
$$

which is a $\operatorname{Seg}(W)$-module. One of our main results (Theorem 3.5) says that if $X$ is irreducible and $H_{D}(X)$ is nonzero, then any irreducible $\operatorname{Seg}(W)$-module in $H_{D}(X)$ determines the central character of $X$. This is an analogue to a statement for Harish-Chandra modules called Vogan's conjecture [HP]. A key step in the proof of Theorem 3.5 is to establish a canonical algebra homomorphism from the center of $\mathcal{H}_{W}$ to the center of $\operatorname{Seg}(W)$ (Theorem 3.4). In the case of the degenerate affine Hecke-Clifford algebra of type $\mathrm{A}_{n-1}$, this homomorphism is shown to map onto the even elements of the center of $\operatorname{Seg}(W)$ via the study of the Dirac cohomology on some modules (Corollary 7.21). The homomorphism indeed agrees with another natural map arising from the Jucys-Murphy type elements (see more detail in Remark 7.22), and hence the property of surjectivity has already been covered in the result of $[\mathrm{Ru}]$.

For a Dirac cohomology in other settings (see, for example, $[\mathrm{HP}]$ ), one may apply the Dirac operator and Dirac cohomology developed in this paper to study the representation theory of $\mathcal{H}_{W}$. More precisely, the action of the Dirac operator provides information about the $\operatorname{Seg}(W)$-module structure and central characters of some $\mathcal{H}_{W}$-modules (see Corollary 4.24 and Theorem 4.25).

We provide evidences that the Dirac cohomology can be useful in the representation theory by computing the action of the Dirac operators in several cases. In Section 5, we consider some basic modules for all classical types and show that the Dirac operator acts identically to zero on those modules. Those modules for type $\mathrm{A}_{n-1}$ were constructed and studied by Hill-Kujawa-Sussan [HKS]. In Section 7, we go further for type $A_{n-1}$ and compute the action of the Dirac type element $D$ on more interesting modules. We show that the Dirac cohomology of those examples does not vanish, and this indeed coincides with the expectation from the case of the degenerate affine Hecke algebra in $[\mathrm{BCT}]$. While some computations 
can also be done for other classical types, the picture is more complete for type $\mathrm{A}_{n-1}$ to date.

This paper is organized as follows. In Section 2, we review some properties of superalgebras. In Section 3, we define a certain algebraic structure $\mathcal{H}_{W}$ and develop the Dirac cohomology theory for $\mathbb{H}_{W}$. We provide examples of $\mathcal{H}_{W}$ in Section 4 and compute the square of the Dirac operator. In Section 5 and Section 7, we consider the Dirac cohomology for some particular modules. In Section 6, we review properties of Sergeev algebra which is needed for the computation of Section 7.

Acknowledgment. The author would like to thank Dan Ciubotaru and Peter Trapa for the suggestion of this topic and many useful discussions. He also thanks Professor Weiqiang Wang for his interest in the work and pointing out the reference [Wa]. The author would also like to thank the referees for useful suggestions and comments, and also thank one of the referees for pointing out the reference $[\mathrm{Ru}]$.

\section{Preliminaries}

\subsection{Notation for modules}

In this paper, all the algebras are associative with a unit over $\mathbb{C}$. Let $\mathcal{A}$ be an algebra. An $\mathcal{A}$-module is denoted $(\pi, X)$ or simply $X$, where $X$ is a vector space and $\pi$ is the map defining the action of $\mathcal{A}$ on $X$. For $a \in \mathcal{A}$ and $x \in X$, the action of $a$ on $x$ is written by $\pi(a) x$ or $a . x$.

Let $\mathcal{B}$ be a subalgebra of $\mathcal{A}$. Define $\operatorname{Ind}_{\mathcal{B}}^{\mathcal{A}}$ to be the induction functor, i.e.,

$$
\operatorname{Ind}_{\mathcal{B}}^{\mathcal{A}} Y=\mathcal{A} \otimes_{\mathcal{B}} Y
$$

where $Y$ is a $\mathcal{B}$-module. The left adjoint functor of $\operatorname{Ind}_{\mathcal{B}}^{\mathcal{A}}$ is the restriction functor denoted $\operatorname{Res}_{\mathcal{B}}^{\mathcal{A}}$.

\subsection{Superalgebras and supermodules}

A super vector space $V$ is a $\mathbb{Z}_{2}$-graded vector space $V=V_{0} \oplus V_{1}$. A super vector subspace $W$ of $V$ is a subspace of $V$ such that $W=\left(W \cap V_{0}\right) \oplus\left(W \cap V_{1}\right)$. We say an element $a$ in $V_{0}\left(\operatorname{resp} . V_{1}\right)$ has even (resp. odd) degree, denoted $\operatorname{deg}(v)=0$ (resp. $\operatorname{deg}(v)=1)$.

A superalgebra $\mathcal{A}$ is an algebra with a super vector space structure $\mathcal{A}=\mathcal{A}_{0} \oplus \mathcal{A}_{1}$ and $\mathcal{A}_{i} \mathcal{A}_{j} \subseteq \mathcal{A}_{i+j}$ for $i, j \in \mathbb{Z}_{2}$. A subalgebra $\mathcal{C}$ of a superalgebra $\mathcal{A}$ is said to be a supersubalgebra of $\mathcal{A}$ if $\mathcal{C}=\left(\mathcal{A}_{0} \cap \mathcal{C}\right) \oplus\left(\mathcal{A}_{1} \cap \mathcal{C}\right)$. A super ideal $\mathcal{I}$ of a superalgebra $\mathcal{A}$ is an ideal of $\mathcal{A}$ such that $\mathcal{I}=\left(\mathcal{A}_{0} \cap \mathcal{I}\right) \oplus\left(\mathcal{A}_{1} \cap \mathcal{I}\right)$.

For superalgebras $\mathcal{A}$ and $\mathcal{B}$, a superalgebra homomorphism from $\mathcal{A}$ to $\mathcal{B}$ is an algebra homomorphism with $f\left(\mathcal{A}_{i}\right) \subset \mathcal{B}_{i}$ for $i \in \mathbb{Z}_{2}$.

For superalgebras $\mathcal{A}$ and $\mathcal{B}$, the super tensor product of $\mathcal{A}$ and $\mathcal{B}$, denoted $\mathcal{A} \widetilde{\otimes} \mathcal{B}$, is a superalgebra isomorphic to $\mathcal{A} \otimes \mathcal{B}$ as vector spaces with the multiplication determined by:

$$
(a \otimes b)\left(a^{\prime} \otimes b^{\prime}\right)=(-1)^{\operatorname{deg}(b) \operatorname{deg}\left(a^{\prime}\right)}\left(a a^{\prime} \otimes b b^{\prime}\right)
$$

where $a, a^{\prime} \in \mathcal{A}$ and $b, b^{\prime} \in \mathcal{B}$ are homogeneous elements. 
Let $\mathcal{A}$ be a superalgebra. An $\mathcal{A}$-supermodule $X$ is an $\mathcal{A}$-module with a super vector space structure $X=X_{0} \oplus X_{1}$ and the property that $\mathcal{A}_{i} . X_{j} \subseteq X_{i+j}$, where $i, j \in \mathbb{Z}_{2}$. A supersubmodule $Y$ of an $\mathcal{A}$-supermodule $X$ is a submodule of $X$ such that $Y=\left(X_{0} \cap Y\right) \oplus\left(X_{1} \cap Y\right)$. An $\mathcal{A}$-supermodule $X$ is irreducible if there is no proper non-zero supersubmodule of $X$.

For an $\mathcal{A}$-supermodule $X=X_{0} \oplus X_{1}$, define a map $\delta: X \rightarrow X$ such that $\delta(v)=v$ if $v \in X_{0}$ and $\delta(v)=-v$ if $v \in X_{1}$.

Let $\operatorname{Mod}_{\text {sup }}(\mathcal{A})$ be the category of $\mathcal{A}$-supermodules. The morphisms in the category $\operatorname{Mod}_{\text {sup }}(\mathcal{A})$ are the even homomorphisms between $\mathcal{A}$-supermodules. Let $\Pi: \operatorname{Mod}_{\text {sup }}(\mathcal{A}) \rightarrow \operatorname{Mod}_{\text {sup }}(\mathcal{A})$ be a parity change functor. That means for an $\mathcal{A}$-supermodule, $\Pi(M)$ and $M$ are isomorphic as $\mathcal{A}$-modules, but have opposite $\mathbb{Z}_{2}$-grading.

\subsection{Relations between irreducible supermodules and irreducible modules}

Let $\mathcal{A}=\mathcal{A}_{0} \oplus \mathcal{A}_{1}$ be a superalgebra. Given an irreducible $\mathcal{A}$-module $(\pi, Y)$, we construct a supermodule as follows. Let $(\bar{\pi}, \bar{Y})$ be an irreducible $\mathcal{A}$-module such that $\bar{Y}$ is identified with $Y$ as vector spaces and the $\mathcal{A}$-action on $\bar{Y}$ is determined for any homogenous element $a \in \mathcal{A}$ and for $v \in Y$ by

$$
\bar{\pi}(a) v=(-1)^{\operatorname{deg}(a)} \pi(a) v .
$$

Let $\left(\pi_{X_{Y}}, X_{Y}\right)$ be an $\mathcal{A}$-supermodule such that $X_{Y}=Y \oplus \bar{Y}$ as vector spaces and the action of $\mathcal{A}$ on $X_{Y}=Y \oplus \bar{Y}$ is as: $\pi_{X_{Y}}(a)(v, \bar{v})=(\pi(a) v, \bar{\pi}(a) \bar{v})$. Let $\left(X_{Y}\right)_{0}=\left\{(v, \bar{v}) \in X_{Y}: v=\bar{v}\right\}$ and let $\left(X_{Y}\right)_{1}=\left\{(v, \bar{v}) \in X_{Y}: v=-\bar{v}\right\}$. It is elementary to check that $X_{Y}=\left(X_{Y}\right)_{0} \oplus\left(X_{Y}\right)_{1}$ is an $\mathcal{A}$-supermodule.

Lemma 2.1. Let $Y$ be an irreducible $\mathcal{A}$-module. Let $X_{Y}=Y \oplus \bar{Y}$ be an $\mathcal{A}$ supermodule with the supermodule structure described above. Then

(1) $X_{Y}$ is an irreducible $\mathcal{A}$-supermodule if and only if $Y$ and $\bar{Y}$ are non-isomorphic as $\mathcal{A}$-modules.

(2) If $Y$ and $\bar{Y}$ are isomorphic as $\mathcal{A}$-modules, then there is a supermodule structure on $Y$.

Proof. For (1), we first prove that if $X_{Y}$ is an irreducible $\mathcal{A}$-supermodule, then $Y$ and $\bar{Y}$ are not isomorphic as $\mathcal{A}$-modules. Suppose instead there exists an $\mathcal{A}$ module isomorphism $f: Y \rightarrow \bar{Y}$, and we will derive a contradiction. Recall that $\bar{Y}$ is identified with $Y$ as vector spaces and thus there exists a natural vector space isomorphism $\theta: \bar{Y} \rightarrow Y$ such that $(-1)^{\operatorname{deg}(a)} \pi(a) \theta=\theta \bar{\pi}(a)$ for any homogenous $a \in \mathcal{A}$. Then $\theta \circ f$ satisfies the property that for any homogenous element $a \in \mathcal{A}$,

$$
\pi(a)(\theta \circ f)(x)=(-1)^{\operatorname{deg}(a)}(\theta \circ f)(\pi(a) x) \quad .
$$

Then the map $(\theta \circ f)^{2}$ is an $\mathcal{A}$-module automorphism of $Y$. Thus, by Schur's lemma and a suitable normalization, we may assume $(\theta \circ f)^{2}$ is an identity map. Then as vector spaces

$$
Y=\operatorname{ker}(\theta \circ f-\mathrm{Id}) \oplus \operatorname{ker}(\theta \circ f+\mathrm{Id}) .
$$


For $\epsilon=0,1$, let

$$
\operatorname{Ker}_{\epsilon}=\left\{\left(v,(-1)^{\epsilon} v\right) \in X_{Y}: v \in \operatorname{ker}\left(\theta \circ f-(-1)^{\epsilon} \mathrm{Id}\right)\right\} .
$$

Then it is straightforward to verify that $\operatorname{Ker}_{0} \oplus \operatorname{Ker}_{1} \subset X_{Y}$ gives a proper supersubmodule of $X_{Y}$.

We now prove that if $Y$ and $\bar{Y}$ are not isomorphic as $\mathcal{A}$-modules, $X_{Y}$ is an irreducible $\mathcal{A}$-supermodule. Suppose instead that there exists a proper supersubmodule $M$ of $X_{Y}$ and we will get a contradiction. Let

$$
M^{i}=\left\{v \in Y:\left(v,(-1)^{i} v\right) \in M \cap\left(X_{Y}\right)_{i}\right\}
$$

for $i \in \mathbb{Z}_{2}$, which are regarded as vector subspaces of $Y$. We first see that $M^{0} \cap$ $M^{1}=0$. Otherwise, there exists some nonzero $v \in Y$ such that $(v, v) \in M$ and $(v,-v) \in M$, and so $(v, 0),(0, v) \in M$. The irreducibility of $Y$ and $\bar{Y}$ implies $M=X_{Y}$, contradicting that $M$ is proper. Furthermore, the irreducibility of $Y$ implies $Y=M^{0} \oplus M^{1}$ (as vector spaces). Define a map $f:(\pi, Y) \rightarrow(\bar{\pi}, \bar{Y})$ determined by $f(v)=(-1)^{i} v$ for $v \in M^{i}\left(i \in \mathbb{Z}_{2}\right)$. One can check that $f$ is an $\mathcal{A}$-module isomorphism and so this gives a contradiction.

We now consider (2). By (1), $X_{Y}$ is not an irreducible $\mathcal{A}$-supermodule. Let $X^{\prime}$ be an irreducible supersubmodule of $X_{Y}$. Then by the construction of $X_{Y}, X^{\prime}$ is isomorphic to $Y=\bar{Y}$ as $\mathcal{A}$-modules. Then this gives a supermodule structure on $Y$.

We can also start with an irreducible $\mathcal{A}$-supermodule and decompose it into irreducible $\mathcal{A}$-module(s).

Lemma 2.2. Let $X$ be an irreducible $\mathcal{A}$-supermodule. Let $\delta$ be a linear automorphism on $X$ such that $\delta(v)=(-1)^{i} v$ for $v \in X_{i}(i=0,1)$. If $X$ is not an irreducible $\mathcal{A}$-module, then there exists an irreducible $\mathcal{A}$-submodule $Y$ of $X$ such that

(1) $\delta(Y)$ is also an $\mathcal{A}$-submodule of $X$ and $\delta(Y)=\bar{Y}$; and

(2) $Y$ and $\delta(Y)$ are non-isomorphic $\mathcal{A}$-modules; and

(3) $X=Y \oplus \delta(Y)$ as $\mathcal{A}$-modules.

Proof. (1) follows from $a . \delta(v)=(-1)^{\operatorname{deg}(a)} \delta(a . v)$ for any homogenous element $a \in \mathcal{A}$ and $v \in Y$. (2) and (3) are (a reformulation of) [BK, Lem. 2.3].

Lemma 2.3. Let $X$ and $X^{\prime}$ be irreducible $\mathcal{A}$-supermodules. If $X$ and $X^{\prime}$ are isomorphic as $\mathcal{A}$-modules, then $X$ and $X^{\prime}$ are isomorphic, up to applying the functor $\Pi$, as $\mathcal{A}$-supermodules.

Proof. Suppose $X$ and $X^{\prime}$ are also irreducible $\mathcal{A}$-modules. Then $X_{0}, X_{1}, X_{0}^{\prime}, X_{1}^{\prime}$ are irreducible $\mathcal{A}_{0}$-modules. Then either $X_{0}=X_{0}^{\prime}$ or $X_{0}=X_{1}^{\prime}$ as $\mathcal{A}_{0}$-modules. Then either $X \cong X^{\prime}$ or $X \cong \Pi\left(X^{\prime}\right)$ as $\mathcal{A}$-supermodules.

Suppose $X$ is not an irreducible $\mathcal{A}$-module. Let $X=Y \oplus \delta(Y)$ and $X^{\prime}=Y^{\prime} \oplus$ $\delta\left(Y^{\prime}\right)$ be the decomposition of $X$ into $\mathcal{A}$-modules as in Lemma 2.2. Without loss of generality, we may assume $Y=Y^{\prime}$ as $\mathcal{A}$-modules. Let $f: Y \rightarrow Y^{\prime}$ be an $\mathcal{A}$-module isomorphism. Then $f$ also induces an $\mathcal{A}$-module isomorphism $\bar{f}: \delta(Y) \rightarrow \delta\left(Y^{\prime}\right)$ such that $\bar{f}=\delta \circ f \circ \delta$. Then one can show that the map $f \oplus \bar{f}$ is an $\mathcal{A}$-supermodule isomorphism by checking that the map preserves grading. In particular, we also have $\Pi(X)=X$ as $\mathcal{A}$-supermodules in this case. 
Let $\operatorname{Irr}(\mathcal{A})\left(\operatorname{resp} . \operatorname{Irr}_{\text {sup }}(\mathcal{A})\right)$ be the set of irreducible $\mathcal{A}$-modules (resp. irreducible $\mathcal{A}$-supermodules). Let $\sim$ be the equivalence relation on $\operatorname{Irr}(\mathcal{A}): Y \sim Y^{\prime}$ if and only if $Y=Y^{\prime}$ or $Y=\overline{Y^{\prime}}$. Let $\sim_{\Pi}$ be the equivalence relation on $\operatorname{Irr}_{\text {sup }}(\mathcal{A})$ : $X \sim_{\Pi} X^{\prime}$ if and only if $X=X^{\prime}$ or $X=\Pi\left(X^{\prime}\right)$.

Proposition 2.4. There is a natural bijection

$$
\operatorname{Irr}_{\text {sup }}(\mathcal{A}) / \sim_{\Pi} \longleftrightarrow \operatorname{Irr}(\mathcal{A}) / \sim .
$$

Proof. Lemmas 2.1 and 2.3 define a map from $\operatorname{Irr}(\mathcal{A}) / \sim$ to $\operatorname{Irr}_{\text {sup }}(\mathcal{A}) / \sim \Pi$. Lemma 2.2 defines a map in the opposite direction. The two maps are inverse to each other by Lemma 2.3 .

\subsection{Central characters of supermodules}

For a superalgebra $\mathcal{A}$, let $Z(\mathcal{A})$ be the center of $\mathcal{A}$. Note that $Z(\mathcal{A})$ is a supersubalgebra of $\mathcal{A}$. Recall that $Z(\mathcal{A})_{0}$ is the set of even elements in $Z(\mathcal{A})$.

Proposition 2.5. Let $X$ be an irreducible $\mathcal{A}$-supermodule. For $z \in Z(\mathcal{A})_{0}$, $z$ acts on $X$ by the multiplication of a scalar.

Proof. If $X$ is an irreducible $\mathcal{A}$-module, then the statement follows from (ordinary) Schur's lemma (for this case). If $X$ is not an irreducible $\mathcal{A}$-module, then we can decompose $X=Y \oplus \delta(Y)$ as $\mathcal{A}$-modules as in Lemma 2.2. Then $z$ acts on the two modules $Y$ and $\delta(Y)$ by scalars, denoted $\lambda$ and $\lambda^{\prime}$ respectively. Then for $v \in Y$,

$$
z \cdot(v+\delta(v))=\frac{\lambda+\lambda^{\prime}}{2}(v+\delta(v))+\frac{\lambda-\lambda^{\prime}}{2}(v-\delta(v)) .
$$

Note that $\delta(v+\delta(v))=v+\delta(v)$ and so $v+\delta(v) \in X_{0}$, and similarly $v-\delta(v) \in X_{1}$. Then since $z$ is of even degree, $\lambda=\lambda^{\prime}$.

By Proposition 2.5, we can define the following:

Definition 2.6. Let $\mathcal{A}$ be a superalgebra. Let $(\pi, X)$ be an irreducible $\mathcal{A}$-supermodule. Define the central character $\chi_{\pi}$ to be the map from $Z(\mathcal{A})_{0}$ to $\mathbb{C}$ such that $\chi_{\pi}(z)$ is the scalar of $z$ acting on $X$.

The central character defined above is only for even elements in the center of a superalgebra. However, the central character indeed determines the action of odd elements in the center in the following sense:

Proposition 2.7. Let $z \in Z(\mathcal{A})_{1}$. Let $X$ be an irreducible $\mathcal{A}$-supermodule. If $X$ is also an irreducible $\mathcal{A}$-module, then $z$ acts by zero on $X$. If $X$ is not an irreducible $\mathcal{A}$-module, then $z$ acts on the two irreducible $\mathcal{A}$-submodules of $X$ by two distinct scalars $\sqrt{\lambda}$ and $-\sqrt{\lambda}$, where $\lambda$ is the scalar that $z^{2} \in Z(\mathcal{A})_{0}$ acts on $X$.

Proof. For (1), suppose $X$ is an irreducible $\mathcal{A}$-module. Then by Schur's Lemma, $z$ acts on $X$ by a scalar denoted by $\lambda$. Meanwhile by Lemmas 2.1 and $2.3, X=\bar{X}$ as $\mathcal{A}$-modules. This implies $z$ also acts by $-\lambda$ on $X$ as $z$ is an odd element. Hence $\lambda=0$.

Now suppose $X$ is not an irreducible $\mathcal{A}$-module. Then $z^{2}$ is an even element in the center and hence acts by a scalar, denoted $\lambda$. Then $z$ acts on the irreducible $\mathcal{A}$-submodules of $X$ by scalars $\sqrt{\lambda}$ and $-\sqrt{\lambda}$. 


\section{Dirac cohomology for $\mathcal{H}_{W}$}

\section{1. $\mathcal{H}_{W}$ and a Dirac type element in $\mathcal{H}_{W}$}

Fix a real reflection group $W$. Let $V$ be a representation of $W$. Fix a $W$-invariant inner product on $V$. Let $\left\{a_{1}, \ldots, a_{n}\right\}$ be an orthogonal basis for $V$.

Definition 3.1. An associative algebra $\mathcal{H}_{W}=\mathcal{H}_{W}(V)$ is said to have property (*) if it satisfies the following properties. First $\mathcal{H}_{W}$ is an algebra generated by symbols $f_{w}(w \in W), c_{i}(i=1, \ldots, n)$ and $a_{i}(i=1, \ldots, n)$ such that the map from $\mathbb{C}[W]$ to $\mathcal{H}_{W}$ sending $w$ to $f_{w}$ is an injection and the algebra has a natural basis of elements having the form $a_{1}^{k_{1}} \cdots a_{n}^{k_{n}} c_{1}^{\epsilon_{1}} \cdots c_{n}^{\epsilon_{n}} f_{w}\left(k_{1}, \ldots, k_{n}\right.$ non-negative integers, $w \in W, \epsilon_{i}=0$ or 1$)$. Again we shall write $w$ for $f_{w}$ for simplicity. Let $\operatorname{Seg}(W)$ be the subalgebra of $\mathcal{H}_{W}$ generated by all $w \in W$ and $c_{i}(i=1, \ldots, n)$. Furthermore, the generators of $\mathcal{H}_{W}$ satisfy the following relations:

$$
\begin{aligned}
w a_{i} w^{-1} & =w\left(a_{i}\right), \\
{\left[a_{i}, a_{j}\right] c_{i} c_{j} } & \in \operatorname{Seg}(W) \quad \text { for } i \neq j, \\
c_{j} a_{i} & =a_{i} c_{j} \quad \text { for } i \neq j, \\
c_{i} a_{i} & =-c_{i} a_{i}, \\
c_{i} c_{j} & =-c_{j} c_{i} \quad \text { for } i \neq j \quad \text { and } c_{i}^{2}=-1, \\
w c_{i} & =w\left(c_{i}\right) w .
\end{aligned}
$$

Here $w\left(a_{i}\right)$ is the action of $w$ on $V$. Furthermore, we identify the linear space spanned by $c_{i}$ with $V$ via the map $a_{i} \mapsto c_{i}$ and hence there is a natural action of $W$ on $c_{i}$, and $w\left(c_{i}\right)$ represents such action of $w$ on $c_{i}$. Indeed, the algebra generated by the those $c_{i}$ is isomorphic to the Clifford algebra on the vector space $V$, and the subalgebra $\operatorname{Seg}(W)$ is the smash product of the Clifford algebra and the group algebra of $W$.

$\mathcal{H}_{W}$ has a superalgebra structure with $\operatorname{deg}\left(c_{i}\right)=1, \operatorname{deg}\left(a_{i}\right)=\operatorname{deg}(w)=0$ $(i=1, \ldots, n$ and $w \in W)$.

In the rest of this section, $\mathcal{H}_{W}$ denotes an algebra satisfying the property $(*)$. Define a Dirac type element $D$ in $\mathcal{H}_{W}$ :

$$
D=\sum_{i=1}^{n} a_{i} c_{i} .
$$

The following two properties will be used several times:

\section{Lemma 3.2.}

(1) $w D=D w$ for any $w \in W$;

(2) $c_{i} D=-D c_{i}$ for any $i$.

Proof. (1) follows from the fact that $\left\{a_{i}\right\}$ forms an orthogonal basis and property (3.1). (2) follows from the properties (3.2), (3.3), and (3.4).

Two homogenous elements $h_{1}, h_{2} \in \mathcal{H}_{W}$ are said to supercommute if $h_{1} h_{2}=$ $(-1)^{\operatorname{deg}\left(h_{1}\right) \operatorname{deg}\left(h_{2}\right)} h_{2} h_{1}$ for any homogenous $w \in \operatorname{Seg}(W)$. 
Definition 3.3. The algebra $\mathcal{H}_{W}$ with the property $(*)$ is said to satisfy the property $(* *)$ if for any $h \in \mathcal{H}_{W}$ such that $h$ supercommutes with elements in $\operatorname{Seg}(W), D^{2} h-h D^{2}=0$.

In the next section, we shall give examples which satisfy the algebraic structure in Definitions 3.1 and 3.3. From now on, assume that $\mathcal{H}_{W}$ satisfies the properties $(*)$ and $(* *)$.

\subsection{Relation between central characters for $\mathcal{H}_{W}$ and $\operatorname{Seg}(W)$}

Let $d: \mathcal{H}_{W} \rightarrow \mathcal{H}_{W}$,

$$
d(h)=D h-(-1)^{\operatorname{deg}(h)} h D .
$$

A relation between $Z\left(\mathcal{H}_{W}\right)_{0}$ and $Z(\operatorname{Seg}(W))_{0}$ is the following:

Theorem 3.4. For any $z \in Z\left(\mathcal{H}_{W}\right)_{0}$, there exists a unique element $\widetilde{z} \in Z(\operatorname{Seg}(W))_{0}$ such that

$$
z-\widetilde{z} \in \operatorname{im} d .
$$

Let $\zeta: Z\left(\mathcal{H}_{W}\right)_{0} \rightarrow Z(\operatorname{Seg}(W))_{0}$ be the map that $\zeta(z)$ is such unique element $\widetilde{z}$ in $Z(\operatorname{Seg}(W))_{0}$. Then $\zeta$ is an algebra homomorphism.

Our main result in this paper is the following, which says the central character of an $\mathcal{H}_{W}$-supermodule $X$ is determined by the central characters of irreducible $\operatorname{Seg}(W)$-supermodules in the Dirac cohomology $H_{D}(X)$. Here $H_{D}(X)$ is defined in the theorem.

Theorem 3.5. Let $\mathcal{H}_{W}$ be an algebra satisfying property $(*)$ (Definition 3.1) and property (**) (Definition 3.3). Let $(\pi, X)$ be an irreducible $\mathcal{H}_{W}$-supermodule with the central character $\chi_{\pi}$ (Definition 2.6). Let the Dirac cohomology $H_{D}(X)$ of $X$ be

$$
H_{D}(X)=\operatorname{ker} \pi(D) /(\operatorname{ker} \pi(D) \cap \operatorname{im} \pi(D)) .
$$

Then $H_{D}(X)$ has a natural $\operatorname{Seg}(W)$-module structure. Let $(\sigma, U)$ be an irreducible $\operatorname{Seg}(W)$-module with the central character $\chi_{\sigma}$ (Definition 2.6) such that

$$
\operatorname{Hom}_{\operatorname{Seg}(W)}\left(U, H_{D}(X)\right) \neq 0 .
$$

Let $\zeta: Z\left(\mathcal{H}_{W}\right)_{0} \rightarrow Z(\operatorname{Seg}(W))_{0}$ be the map in Theorem 3.4. Let $\chi^{\sigma}: Z\left(\mathcal{H}_{W}\right)_{0} \rightarrow \mathbb{C}$,

$$
\chi^{\sigma}(z)=\chi_{\sigma}(\zeta(z)) .
$$

Then $\chi_{\pi}=\chi^{\sigma}$.

Since $w D=D w$ and $c_{i} D=-D c_{i}$ by Lemma $3.2, \operatorname{ker} \pi(D)$ and $\operatorname{ker} \pi(D) \cap$ $\operatorname{im} \pi(D)$ are invariant under the action of $\operatorname{Seg}(W)$. Thus $H_{D}(X)$ has a natural $\operatorname{Seg}(W)$-module structure from the $\mathcal{H}_{W}$-module structure. The proofs of Theorems 3.4 and 3.5 are given at the end of the next subsection. Theorem 3.5 directly follows from Theorem 3.4. Readers who only want to know how Theorem 3.4 implies Theorem 3.5 may jump to the end of the next subsection. 


\subsection{Proof of Theorems 3.4 and 3.5}

The proofs of the theorems basically follow from the ideas of proofs in [HP, Chap. $3]$ and [BCT, Sect. 4]. We provide some technical details for this specific case.

Let $S \leq j(V)$ be the vector space of polynomials of $x_{1}, \ldots, x_{n}$ with degree less than or equal to $j$. Let $\mathcal{H}_{W}^{j}$ be the vector space spanned by elements of the form

$$
\left\{p w: w \in \operatorname{Seg}(W), p \in S^{\leq j}(V)\right\}
$$

Note that $\mathcal{H}_{W}^{0} \subseteq \mathcal{H}_{W}^{1} \subseteq \cdots$ gives a filtration for $\mathcal{H}_{W}$. Define

$$
\overline{\mathcal{H}}_{W}^{r}=\mathcal{H}_{W}^{r} / \mathcal{H}_{W}^{r-1}
$$

for $r=0,1, \ldots$ and $\mathcal{H}_{W}^{-1}=0$. Let $\overline{\mathcal{H}}_{W}=\bigoplus_{j=0}^{\infty} \overline{\mathcal{H}}_{W}^{j}$. Note that $\overline{\mathcal{H}}_{W}$ has a natural superalgebra structure from $\mathcal{H}_{W}$.

Let $d_{j}: \overline{\mathcal{H}}_{W}^{j} \rightarrow \overline{\mathcal{H}}_{W}^{j+1}$ be the map induced from $d$ and let $\bar{d}=\bigoplus_{j=0}^{\infty} d_{j}$. For any element $h \in \mathcal{H}_{W}$, we still write $h$ for its corresponding element in $\overline{\mathcal{H}}_{W}$. Let $b_{i}=a_{i} c_{i}(i=1, \ldots, n)$. Let $\mathcal{B}$ be the supersubalgebra of $\overline{\mathcal{H}}_{W}$ generated by all $b_{i}$. Note that $\bar{d}(\mathcal{B}) \subset \mathcal{B}$. Let $\bar{d}^{\prime}$ be the restriction of $\bar{d}$ to $\mathcal{B}$.

In the following lemmas, one can see that $\operatorname{ker} \bar{d}^{\prime}, \operatorname{im} \bar{d}^{\prime}, \operatorname{ker} \bar{d},(\operatorname{ker} d \cap \operatorname{im} d)^{\operatorname{Seg}(W)}$ and so on are supersubspaces by using the fact that $D$ is an homogenous element.

Lemma 3.6. As supersubspaces of $\mathcal{B}$,

$$
\operatorname{ker} \bar{d}^{\prime}=\operatorname{im} \bar{d}^{\prime} \oplus \mathbb{C} .
$$

Here $\mathbb{C}$ is regarded as the $\mathbb{C}$-subalgebra of $\mathcal{B}$ generated by 1 .

Proof. Note that any element in $\mathcal{B}$ can be uniquely written as a linear combination of elements of the form $p b_{i_{1}} b_{i_{2}} \ldots b_{i_{r}}$ for $0<i_{1}<\ldots<i_{r} \leq n$ and $p \in \mathbb{C}\left[b_{1}^{2}, \ldots, b_{n}^{2}\right]$. Note that $D=\sum_{i=1}^{n} b_{i}$. Using the relations $b_{i} b_{j}=-b_{j} b_{i}$ (in $\mathcal{B}$ ) for $i \neq j$ and $b_{i}^{2} b_{j}=b_{j} b_{i}^{2}($ in $\mathcal{B})$ for any $i, j$, one can see that the action of $\bar{d}^{\prime}$ is determined by

$$
\bar{d}^{\prime}\left(p b_{i_{1}} b_{i_{2}} \ldots b_{i_{r}}\right)=2 \sum_{k=1}^{r}(-1)^{k-1} b_{i_{k}}^{2} p b_{i_{1}} \ldots \widehat{b_{i_{k}}} \ldots b_{i_{r}}
$$

where $p \in \mathbb{C}\left[b_{1}^{2}, \ldots, b_{n}^{2}\right]$.

In order to apply the known cohomology of the Koszul complex, we identify $\mathcal{B}$ with $\mathbb{C}\left[x_{1}, \ldots, x_{n}\right] \otimes \wedge^{\bullet} \mathbb{C}^{n}$ as vector spaces, where $\wedge^{\bullet} \mathbb{C}^{n}$ is the exterior algebra, via the linear isomorphism $\eta$ from $\mathbb{C}\left[x_{1}, \ldots, x_{n}\right] \otimes \wedge^{\bullet} \mathbb{C}^{n}$ to $\mathcal{B}$ determined by

$$
\eta: p\left(x_{1}, \ldots, x_{n}\right) \otimes e_{i_{1}} \wedge \ldots \wedge e_{i_{k}} \mapsto p\left(b_{1}^{2}, \ldots, b_{n}^{2}\right) b_{i_{1}} \ldots b_{i_{k}}
$$

where $\left\{e_{1}, \ldots, e_{n}\right\}$ is the standard basis of $\mathbb{C}^{n}$. Then, by the above description of the action of $\bar{d}^{\prime}$, the map $\eta^{-1} \circ \bar{d}^{\prime} \circ \eta$ is a multiple of the differential map in the standard Koszul resolution. Then the result follows from the well known cohomology of the Koszul resolution. 
Proposition 3.7. As supersubspaces of $\overline{\mathcal{H}}_{W}$,

$$
\operatorname{ker} \bar{d}=\operatorname{im} \bar{d} \oplus \operatorname{Seg}(W) .
$$

Proof. By the property $(*)$ of $\mathcal{H}_{W}, a_{1}^{m_{1}} a_{2}^{m_{2}} \cdots a_{n}^{m_{n}} c_{1}^{\epsilon_{1}} \cdots c_{n}^{\epsilon_{n}} w\left(m_{i} \in \mathbb{Z}_{\geq 0}, \epsilon_{i} \in\right.$ $\{0,1\}$ and $w \in W)$ form a basis for $\overline{\mathcal{H}}_{W}$. Then $b_{1}^{m_{1}} b_{2}^{m_{2}} \cdots b_{n}^{m_{n}} c_{1}^{\epsilon_{1}} \cdots c_{n}^{\epsilon_{n}} w\left(m_{i} \in\right.$ $\mathbb{Z}_{\geq 0}, \epsilon_{i} \in\{0,1\}$ and $w \in W$ ) also form a basis for $\overline{\mathcal{H}}_{W}$. Then as linear vector spaces, we may identify $\overline{\mathcal{H}}_{W}$ with $\mathcal{B} \otimes \operatorname{Seg}(W)$ via the following map:

$$
b_{1}^{m_{1}} b_{2}^{m_{2}} \cdots b_{n}^{m_{n}} c_{1}^{\epsilon_{1}} \cdots c_{n}^{\epsilon_{n}} w \mapsto b_{1}^{m_{1}} \cdots b_{n}^{m_{n}} \otimes c_{1}^{\epsilon_{1}} \cdots c_{n}^{\epsilon_{n}} w .
$$

For any $h \in \overline{\mathcal{H}}_{W}, \bar{d}(h w)=\bar{d}(h) w$ for $w \in W$ and $\bar{d}\left(h c_{i}\right)=\bar{d}(h) c_{i}$. Then the map $\bar{d}$ in $\mathcal{H}_{W}$ is the same as $\bar{d}^{\prime} \otimes \operatorname{Id}$ in $\mathcal{B} \otimes \operatorname{Seg}(W)$ under the above identification. Then by Lemma 3.6, one has

$$
\begin{aligned}
\operatorname{ker} \bar{d} & =\operatorname{ker}\left(\bar{d}^{\prime} \otimes \mathrm{Id}\right)=\left(\operatorname{ker} \bar{d}^{\prime}\right) \otimes \operatorname{Seg}(W) \\
& =\left(\operatorname{im} \bar{d}^{\prime} \oplus \mathbb{C}\right) \otimes \operatorname{Seg}(W)=\operatorname{im} \bar{d} \oplus \operatorname{Seg}(W) .
\end{aligned}
$$

For any subspace $H$ of $\mathcal{H}_{W}$, define $H^{\operatorname{Seg}(W)}$ to be the set of all elements supercommuting with elements in $\operatorname{Seg}(W)$. If we view $\operatorname{Seg}(W)$ as a subalgebra of $\overline{\mathcal{H}}_{W}$, we could similarly define $\bar{H}^{\operatorname{Seg}(W)}$ for any subspace $\bar{H}$ of $\overline{\mathcal{H}}_{W}$. Proposition 3.7 implies the following:

Corollary 3.8. As supersubspaces of $\overline{\mathcal{H}}_{W}$,

$$
(\operatorname{ker} \bar{d})^{\operatorname{Seg}(W)}=(\operatorname{im} \bar{d})^{\operatorname{Seg}(W)} \oplus Z(\operatorname{Seg}(W)) .
$$

Lemma 3.9. As supersubspaces of $\mathcal{H}_{W}$,

$$
(\operatorname{ker} d)^{\operatorname{Seg}(W)}=(\operatorname{ker} d \cap \operatorname{im} d)^{\operatorname{Seg}(W)} \oplus Z(\operatorname{Seg}(W)) .
$$

Proof. It is clear that $Z(\operatorname{Seg}(W))$ and $(\operatorname{ker} d \cap i m d)^{\operatorname{Seg}(W)}$ are subspaces of the space $(\operatorname{ker} d)^{\operatorname{Seg}(W)}$ and thus $(\operatorname{ker} d \cap \operatorname{im} d)^{\operatorname{Seg}(W)} \oplus Z(\operatorname{Seg}(W)) \subset(\operatorname{ker} d)^{\operatorname{Seg}(W)}$. We will prove another inclusion by induction on the degree of filtration of an element in $(\operatorname{ker} d)^{\operatorname{Seg}(W)}$.

Let $h$ be an element in $(\operatorname{ker} d)^{\operatorname{Seg}(W)}$ such that $h \in \mathcal{H}_{W}^{i}$ and $h \notin \mathcal{H}_{W}^{i-1}$ for some $i$. When $i=0, \mathcal{H}_{W}^{0}=\operatorname{Seg}(W)$ and so the statement is clearly true. Now assume $i>0$. Let $\bar{h}$ be the image of $h$ in $\overline{\mathcal{H}}_{W}^{i}$. Then by Corollary $3.8, \bar{h}=\bar{d}\left(\bar{h}_{0}\right)$ for some unique $\bar{h}_{0}$ in $\overline{\mathcal{H}}_{W}^{i-1}$ such that $\bar{d}\left(\bar{h}_{0}\right) \in\left(\overline{\mathcal{H}}_{W}^{i}\right)^{\operatorname{Seg}(W)}$. For any representative $h_{0}^{\prime} \in \mathcal{H}_{W}^{i-1}$ of $\bar{h}_{0}$, let

$$
h_{0}=\frac{1}{2^{n}|W|} \sum_{k=1}^{n} \sum_{i_{1}<\ldots<i_{k}} \sum_{w \in W}(-1)^{k}\left(c_{i_{1}} \cdots c_{i_{k}}\right) w h_{0}^{\prime} w^{-1}\left(c_{i_{1}} \cdots c_{i_{k}}\right)^{-1} .
$$

By the uniqueness of the element $\bar{h}_{0}, \bar{h}_{0}$ supercommutes with any element in $\operatorname{Seg}(W)$. This implies $h_{0}$ is also a representative of $\bar{h}_{0}$. Furthermore, $h_{0}$ supercommutes with elements in $\operatorname{Seg}(W)$ and $d\left(h_{0}\right) \in\left(\mathcal{H}_{W}^{i}\right)^{\operatorname{Seg}(W)}$. By the property $(* *)$, $d^{2}\left(h_{0}\right)=0$ and so $d\left(h-d\left(h_{0}\right)\right)=0$. By the induction hypothesis, $h-d\left(h_{0}\right) \in$ $(\operatorname{im} d)^{\operatorname{Seg}(W)} \oplus Z(\operatorname{Seg}(W))$. Hence, we also have $h \in(\operatorname{im} d)^{\operatorname{Seg}(W)} \oplus Z(\operatorname{Seg}(W))$ since $d\left(h_{0}\right) \in(\operatorname{im} d)^{\operatorname{Seg}(W)}$. This completes the proof. 
Lemma 3.10. $(\operatorname{ker} d)^{\operatorname{Seg}(W)}$ is a supersubalgebra of $\mathcal{H}_{W}$ and $(\operatorname{ker} d \cap \operatorname{im} d)^{\operatorname{Seg}(W)}$ is a two-sided super ideal of $(\operatorname{ker} d)^{\operatorname{Seg}(W)}$.

Proof. Let $z_{1}, z_{2} \in(\operatorname{ker} d)^{\operatorname{Seg}(W)}$. Then $d\left(z_{i}\right)=0$ and so $D z_{i}=\delta\left(z_{i}\right) D$. Now $d\left(z_{1} z_{2}\right)=D\left(z_{1} z_{2}\right)-\delta\left(z_{1} z_{2}\right) D=\delta\left(z_{1} z_{2}\right) D-\delta\left(z_{1} z_{2}\right) D=0$. Hence $z_{1} z_{2} \in$ $(\operatorname{ker} d)^{\operatorname{Seg}(W)}$. Hence $(\operatorname{ker} d)^{\operatorname{Seg}(W)}$ is a subalgebra of $\mathcal{H}_{W}$.

We next show that $(\operatorname{ker} d \cap \operatorname{im} d)^{\operatorname{Seg}(W)}$ is a two-sided ideal of $(\operatorname{ker} d)^{\operatorname{Seg}(W)}$. Let $z \in(\operatorname{ker} d)^{\operatorname{Seg}(W)}$ and $z^{\prime} \in(\operatorname{ker} d \cap \operatorname{im} d)^{\operatorname{Seg}(W)}$. We have to show $z z^{\prime}, z^{\prime} z \in$ $(\operatorname{ker} d \cap \operatorname{im} d)^{\operatorname{Seg}(W)}$. Write $z^{\prime}=D h-\delta(h) D$ for some $h \in \mathcal{H}_{W}$. Since $d(z)=$ $D z-\delta(z) D=0$

$$
z z^{\prime}=z D h-z \delta(h) D=D \delta(z) h-z \delta(h) D=D \delta(z) h-\delta(z \delta(h)) D \in \operatorname{im} d .
$$

We also proved in the beginning that $z z^{\prime} \in \operatorname{ker} d$ and thus $z z^{\prime} \in(\operatorname{ker} d \cap \operatorname{im} d)^{\operatorname{Seg}(W)}$. The proof for $z^{\prime} z \in(\operatorname{ker} d \cap \operatorname{im} d)^{\operatorname{Seg}(W)}$ is similar.

Proof of Theorem 3.4 Since $z \in Z\left(\mathcal{H}_{W}\right)_{0} \subset \operatorname{ker} d^{\operatorname{Seg}(W)}$, by Lemma 3.9, there exists a unique $\widetilde{z} \in Z(\operatorname{Seg}(W))$ such that $z-\widetilde{z} \in(\operatorname{ker} d \cap \operatorname{im} d)^{\operatorname{Seg}(W)} \subset \operatorname{im} d$. Note that $\widetilde{z}$ is in $Z(\operatorname{Seg}(W))_{0}$ since the decomposition in Lemma 3.9 is between super vector spaces. Hence we have a $\operatorname{map} \zeta: Z\left(\mathcal{H}_{W}\right)_{0} \rightarrow Z(\operatorname{Seg}(W))_{0}$.

It remains to prove that $\zeta$ is an algebra homomorphism. To see that $\zeta$ is an algebra map, let $z_{i} \in Z\left(\mathcal{H}_{W}\right) \subset \operatorname{ker} d^{\operatorname{Seg}(W)}(i=1,2)$. Write $z_{i}=\zeta\left(z_{i}\right)+h_{i}$ for some $h_{i} \in(\operatorname{ker} d \cap \operatorname{im} d)^{\operatorname{Seg}(W)}$. Then $z_{1} z_{2}=\zeta\left(z_{1}\right) \zeta\left(z_{2}\right)+\zeta\left(z_{1}\right) h_{2}+\zeta\left(z_{2}\right) h_{1}+$ $h_{1} h_{2}$. By Lemma 3.10, $z_{1} z_{2}-\zeta\left(z_{1}\right) \zeta\left(z_{2}\right) \in(\operatorname{ker} d \cap \operatorname{im} d)^{\operatorname{Seg}(W)}$. Thus $\zeta\left(z_{1} z_{2}\right)=$ $\zeta\left(z_{1}\right) \zeta\left(z_{2}\right)$. This completes the proof.

Proof of Theorem 3.5. By our hypothesis, there exists a non-zero element $v \in$ $H_{D}(X)$ such that $v$ is in the isotypic component $U$ of $H_{D}(X)$. Let $\widetilde{v}$ be a representative of $v$ in $\operatorname{ker} \pi(D)$. Now by Theorem 3.4 for any $z \in Z\left(\mathcal{H}_{W}\right)_{0}$, $z-\zeta(z)=D a-\delta(a) D$ for some $a \in \mathcal{H}_{W}$. Then $\pi(z-\zeta(z)) \widetilde{v}=\pi(D a-\delta(a) D) \widetilde{v}=$ $\pi(D a) \widetilde{v} \in \operatorname{im} \pi(D)$. On the other hand, $\pi(z-\zeta(z)) \widetilde{v}=\chi_{\pi}(z) \widetilde{v}-\left(\chi_{\sigma}(\zeta(z)) \widetilde{v}+\widetilde{v}^{\prime}\right)$ for some $\widetilde{v}^{\prime} \in \operatorname{ker} \pi(D) \cap \operatorname{im} \pi(D)$ and so $\left(\chi_{\pi}(z)-\chi_{\sigma}(\zeta(z))\right) \widetilde{v} \in \operatorname{im} \pi(D)$. We also have $\left(\chi_{\pi}(z)-\chi_{\sigma}(\zeta(z))\right) \widetilde{v} \in \operatorname{ker} \pi(D)$ as $\widetilde{v} \in \operatorname{ker} \pi(D)$. Thus $\chi_{\pi}(z) \widetilde{v}-\chi_{\sigma}(\zeta(z)) \widetilde{v} \in$ $\operatorname{im} \pi(D) \cap \operatorname{ker} \pi(D)$. Since we choose $v \neq 0$, we can only have $\chi_{\pi}(z)=\chi_{\sigma}(\zeta(z))=$ $\chi^{\sigma}(z)$. This completes the proof.

\section{Examples of $\mathcal{H}_{W}$ and their Dirac cohomology theory}

Let $W$ be a classical Weyl group and let $R=R(W)$ be the root system associated to $W$. Let $\mathbf{k}: R \rightarrow \mathbb{C}$ be a function such that $\mathbf{k}\left(\alpha_{1}\right)=\mathbf{k}\left(\alpha_{2}\right)$ if $\alpha_{1}=w\left(\alpha_{2}\right)$ for some $w \in W$. We shall write $\mathbf{k}_{\alpha}$ for $\mathbf{k}(\alpha)$. For any $\alpha \in R$, let $s_{\alpha}$ be the simple reflection associated to $\alpha$.

Let $e_{1}, \ldots, e_{n}$ be the standard basis of $\mathbb{R}^{n}$. Let $\langle$,$\rangle be the inner product on \mathbb{R}^{n}$ such that $\left\langle e_{i}, e_{j}\right\rangle=\delta_{i j}$.

\subsection{Type $A_{n-1}$}

Notation 4.1. Set $W=W\left(\mathrm{~A}_{n-1}\right)$ to be the Weyl group of type $\mathrm{A}_{n-1}$. The root system $R\left(\mathrm{~A}_{n-1}\right)$ of type $\mathrm{A}_{n-1}$ is the set

$$
R\left(\mathrm{~A}_{n-1}\right)=\left\{e_{i}-e_{j}: 1 \leq i \neq j \leq n\right\} .
$$


Fix a set $R^{+}$of positive roots

$$
R^{+}\left(\mathrm{A}_{n-1}\right)=\left\{e_{i}-e_{j}: 1 \leq i<j \leq n\right\}
$$

We usually write $\alpha>0$ for $\alpha \in R^{+}\left(\mathrm{A}_{n-1}\right)$ and write $\alpha<0$ for $-\alpha \in R^{+}\left(\mathrm{A}_{n-1}\right)$. The set of simple roots $\Delta$ is

$$
\left\{e_{i}-e_{i+1}: i=1, \ldots, n-1\right\} .
$$

Since there is only one $W$-orbit for $R\left(\mathrm{~A}_{n-1}\right)$, we simply write $\mathbf{k}$ for $\mathbf{k}_{\alpha}$ for any $\alpha \in R\left(\mathrm{~A}_{n-1}\right)$. For $i \neq j$, let

$$
\alpha_{i j}= \begin{cases}e_{i}-e_{j} & \text { if } i<j \\ e_{j}-e_{i} & \text { if } i>j\end{cases}
$$

Thus $\alpha_{i j}$ is always a positive root.

For a root $\alpha \in R\left(\mathrm{~A}_{n-1}\right)$, let $s_{\alpha}$ be the corresponding simple reflection in $W\left(\mathrm{~A}_{n-1}\right)$. For simplicity, set $s_{i j}=s_{\alpha_{i j}}$.

Definition 4.2 ([Na]). The degenerate affine Hecke-Clifford algebra for type $A_{n-1}$, denoted $\mathbb{H}_{W\left(\mathrm{~A}_{n-1}\right)}^{\mathrm{Cl}}$, is the associative algebra with a unit generated by the symbols $\left\{x_{i}\right\}_{i=1}^{n},\left\{c_{i}\right\}_{i=1}^{n}$ and $\left\{f_{w}: w \in W\left(\mathrm{~A}_{n-1}\right)\right\}$ determined by the following properties:

(1) the map from the group algebra $\mathbb{C}\left[W\left(\mathrm{~A}_{n-1}\right)\right]=\bigoplus_{w \in W\left(\mathrm{~A}_{n-1}\right)} \mathbb{C} w$ to $\mathbb{H}_{W\left(\mathrm{~A}_{n-1}\right)}^{\mathrm{Cl}}$ given by $w \mapsto f_{w}$ is an algebra injection;

(2) $x_{i} x_{j}=x_{j} x_{i}$ for all $i, j$;

(3) $x_{i} c_{j}=c_{j} x_{i}$ for $i \neq j$ and $x_{i} c_{i}=-c_{i} x_{i}$ for all $i$;

(4) $c_{i} c_{j}=-c_{j} c_{i}$ for $i \neq j$ and $c_{i}^{2}=-1$ for all $i$;

(5) $f_{w} c_{i}=c_{w(i)} f_{w}$ for $w \in W\left(\mathrm{~A}_{n-1}\right)$ and for all $i$;

(6) $f_{s_{i, i+1}} x_{i}-x_{i+1} f_{s_{i, i+1}}=\mathbf{k}\left(-1+c_{i} c_{i+1}\right)$ for all $i=1, \ldots, n-1$ and $f_{s_{i, i+1}} x_{j}=$ $x_{j} f_{s_{i, i+1}}$ for all $i, j$ with $|i-j|>1$.

We later simply write $w$ for $f_{w}$. The algebra has a superalgebra structure with $\operatorname{deg}\left(c_{i}\right)=1, \operatorname{deg}(w)=0$ for $w \in W\left(\mathrm{~A}_{n-1}\right)$, and $\operatorname{deg}\left(x_{i}\right)=0$.

For $i \neq j$, define $c_{\alpha_{i j}}$ as

$$
c_{\alpha_{i j}}= \begin{cases}\frac{\sqrt{2}}{2}\left(c_{i}-c_{j}\right) & \text { if } i<j \\ \frac{\sqrt{2}}{2}\left(c_{j}-c_{i}\right) & \text { if } j<i\end{cases}
$$

Let $\widetilde{s}_{\alpha_{i j}}=\widetilde{s}_{i j}=s_{i j} c_{\alpha_{i j}}$.

The superalgebra $\mathbb{H}_{W\left(\mathrm{~A}_{n-1}\right)}^{\mathrm{Cl}}$ admits a PBW type basis:

Proposition 4.3 ([Kl, Thm. 14.2.2]). The set

$$
\left\{x_{1}^{m_{1}} \cdots x_{n}^{m_{n}} c_{1}^{\epsilon_{1}} \cdots c_{n}^{\epsilon_{n}} w: m_{1}, \ldots, m_{n} \in \mathbb{Z}_{\geq 0}, \epsilon_{1}, \ldots, \epsilon_{n} \in\{0,1\}, w \in W\left(\mathrm{~A}_{n-1}\right)\right\}
$$

forms a basis for $\mathbb{H}_{W\left(\mathrm{~A}_{n-1}\right)}^{\mathrm{Cl}}$.

The main statement of this subsection is Proposition 4.9, which says that $\mathbb{H}_{W\left(\mathrm{~A}_{n-1}\right)}^{\mathrm{Cl}}$ satisfies property $(*)$ defined in Definition 3.1. 
Let $\widetilde{s}_{\alpha}=s_{\alpha} c_{\alpha}$. For later convenience, we also set $\widetilde{s}_{i j}=\widetilde{s}_{\alpha_{i j}}=s_{\alpha_{i j}} c_{\alpha_{i j}}, y_{i}=$ $x_{i} c_{i}, y_{i}^{\prime}=y_{i}+\frac{\sqrt{2}}{2} \sum_{i \neq j} \widetilde{s}_{i, j}$ and $x_{i}^{\prime}=-y_{i}^{\prime} c_{i}$. Note that $\mathbb{C}\left[W\left(\mathrm{~A}_{n-1}\right)\right]^{-}$embeds into $\mathbb{H}_{W\left(\mathrm{~A}_{n-1}\right)}^{\mathrm{Cl}}$ via the map $\widetilde{t}_{\alpha} \mapsto \widetilde{s}_{\alpha}$.

The notations $y_{i}^{\prime}$ and $x_{i}^{\prime}$ will be used to define the Dirac type element in $\mathbb{H}_{W\left(\mathrm{~A}_{n-1}\right)}^{\mathrm{Cl}}$ and are inspired by the setting in the degenerate affine Hecke algebra in [BCT].

\section{Lemma 4.4.}

(1) $c_{i} y_{j}=-y_{j} c_{i}$ for any $i, j$;

(2) $\widetilde{s}_{i j} c_{k}=-c_{k} \widetilde{s}_{i j}$ for any $i, j, k$ with $i \neq j$;

(3) $c_{i} y_{j}^{\prime}=-y_{j}^{\prime} c_{i}$ for any $i, j$;

(4) For $\alpha \in R^{+}$and $w \in S_{n}, w \widetilde{s}_{\alpha} w^{-1}=\widetilde{s}_{w(\alpha)}$ if $w(\alpha)>0$, and $w \widetilde{s}_{\alpha} w^{-1}=$ $-\widetilde{s}_{-w(\alpha)}$ if $w(\alpha)<0$.

The above lemma is elementary. We skip the proof.

We shall use the natural permutation of $W\left(\mathrm{~A}_{n-1}\right)$ on the set $\{1, \ldots, n\}$ below.

Lemma 4.5. Let $w \in W\left(\mathrm{~A}_{n-1}\right)$. Then

$$
w y_{i} w^{-1}-y_{w(i)}=\sqrt{2} \mathbf{k} \sum_{\beta>0, w^{-1}(\beta)<0,\left\langle\beta, w\left(e_{i}\right)\right\rangle \neq 0} \widetilde{s}_{\beta} .
$$

In particular, for $\alpha>0$,

$$
\widetilde{s}_{\alpha} y_{i} \widetilde{s}_{\alpha}^{-1}+y_{s_{\alpha}(i)}=-\sqrt{2} \mathbf{k} \sum_{\beta>0, s_{\alpha}^{-1}(\beta)<0,\left\langle\beta, s_{\alpha}\left(e_{i}\right)\right\rangle \neq 0} \widetilde{s}_{\beta} .
$$

Proof. For $w \in W\left(\mathrm{~A}_{n-1}\right)$, define $l(w)=\left|\left\{e_{i}-e_{j} \in R^{+}\left(\mathrm{A}_{n-1}\right): w\left(e_{i}-e_{j}\right)<0\right\}\right|$. When $l(w)=1, w=s_{\alpha}$ for some $\alpha \in \Delta$. We consider three cases. When $\left\langle e_{i}, \alpha\right\rangle=0$, it is easy to see $s_{\alpha} y_{i} s_{\alpha}-y_{i}=0$. Now consider the case $\left\langle e_{i}, \alpha\right\rangle=1$. In this case, we have

$$
\begin{aligned}
s_{\alpha} y_{i} s_{\alpha} & =s_{\alpha} x_{i} c_{i} s_{\alpha} \\
& =x_{i+1} c_{i+1}+\mathbf{k}\left(-1+c_{i} c_{i+1}\right) c_{i} s_{\alpha} \\
& =x_{i+1} c_{i+1}+\mathbf{k}\left(-c_{i}+c_{i+1}\right) s_{\alpha} \\
& =x_{i+1} c_{i+1}+\mathbf{k} s_{\alpha}\left(c_{i}-c_{i+1}\right) \\
& =y_{i+1}+\sqrt{2} \mathbf{k} \widetilde{s}_{\alpha} .
\end{aligned}
$$

For $\left\langle e_{i}, \alpha\right\rangle=-1$, by using $s_{\alpha} \widetilde{s}_{\alpha} s_{\alpha}=-\widetilde{s}_{\alpha}$ and the computation in the case $\left\langle e_{i}, \alpha\right\rangle=1$, we have

$$
s_{\alpha} y_{i+1} s_{\alpha}=y_{i}+\sqrt{2} \mathbf{k} \widetilde{s}_{\alpha} .
$$

We now use an induction on $l(w)$. Assume $l(w)=k$ for some $k>1$. Write $w=s_{\alpha} w^{\prime}$ for some simple reflection $s_{\alpha}$ and $w^{\prime} \in W\left(\mathrm{~A}_{n-1}\right)$ with $l\left(w^{\prime}\right)=k-1$. Set 
$\epsilon=1$ if $\left\langle\alpha, w\left(e_{i}\right)\right\rangle \neq 0$ and $\epsilon=0$ otherwise. Then

$$
\begin{aligned}
w y_{i} w^{-1} & =s_{\alpha} w^{\prime} y_{i} w^{\prime-1} s_{\alpha} \\
& =s_{\alpha} y_{w^{\prime}(i)} s_{\alpha}+\sqrt{2} \mathbf{k} \sum_{\beta>0, w^{\prime-1}(\beta)<0,\left\langle\beta, w^{\prime}\left(e_{i}\right)\right\rangle \neq 0} s_{\alpha} \widetilde{s}_{\beta} s_{\alpha} \quad \text { (induction hypothesis) } \\
& \left.=y_{s_{\alpha} w^{\prime}(i)}+\epsilon \sqrt{2} \mathbf{k} \widetilde{s}_{\alpha}+\sqrt{2} \mathbf{k} \sum_{\beta>0, w^{\prime-1}} \widetilde{s}_{(\beta)<0,\left\langle\beta, w^{\prime}\left(e_{i}\right)\right\rangle \neq 0} \quad \text { (calculation for } l(w)=1\right) \\
& =y_{s_{\alpha} w^{\prime}(i)}+\epsilon \sqrt{2} \mathbf{k} \widetilde{s}_{\alpha}+\sqrt{2} \mathbf{k} \sum_{\beta>0, w^{\prime-1}(\beta)<0,\left\langle s_{\alpha}(\beta), s_{\alpha} w^{\prime}\left(e_{i}\right)\right\rangle \neq 0} \widetilde{s}_{s_{\alpha}(\beta)} \\
& =y_{w(i)}+\sqrt{2} \mathbf{k} \sum_{\beta>0, w^{-1}(\beta)<0,\left\langle\beta, w\left(e_{i}\right)\right\rangle \neq 0} \widetilde{s}_{\beta} .
\end{aligned}
$$

This proves the first assertion. The second assertion follows from the first one with the equation that

$$
\widetilde{s}_{\alpha} y_{i} \widetilde{s}_{\alpha}^{-1}=s_{\alpha} c_{\alpha} y_{i}\left(-c_{\alpha} s_{\alpha}\right)=s_{\alpha}\left(c_{\alpha}^{2}\right) y_{i} s_{\alpha}=-s_{\alpha} y_{i} s_{\alpha}
$$

Lemma 4.6. For $i \neq j,\left[x_{i}^{\prime}, x_{j}^{\prime}\right] c_{i} c_{j}=y_{i}^{\prime} y_{j}^{\prime}+y_{j}^{\prime} y_{i}^{\prime} \in \operatorname{Seg}_{n}$.

Proof.

$$
\begin{aligned}
y_{i}^{\prime} y_{j}^{\prime}+y_{j}^{\prime} y_{i}^{\prime}= & \left(y_{i}+\frac{\sqrt{2}}{2} \mathbf{k} \sum_{k \neq i} \widetilde{s}_{i, k}\right)\left(y_{j}+\mathbf{k} \frac{\sqrt{2}}{2} \sum_{l \neq j} \widetilde{s}_{l, j}\right) \\
& +\left(y_{j}+\frac{\sqrt{2}}{2} \mathbf{k} \sum_{l \neq j} \widetilde{s}_{l, j}\right)\left(y_{i}+\frac{\sqrt{2}}{2} \mathbf{k} \sum_{k \neq i} \widetilde{s}_{i, k}\right) \\
= & y_{i} y_{j}+y_{j} y_{i}+\frac{\sqrt{2}}{2} \mathbf{k}\left(\sum_{k \neq i} \widetilde{s}_{i, k} y_{j}+y_{j} \sum_{k \neq i} \widetilde{s}_{i, k}+y_{i} \sum_{l \neq j} \widetilde{s}_{l, j}+\sum_{l \neq j} \widetilde{s}_{l, j} y_{i}\right) \\
& +\frac{1}{2} \mathbf{k}^{2}\left(\sum_{k \neq i} \widetilde{s}_{i, k} \sum_{l \neq j} \widetilde{s}_{l, j}+\sum_{l \neq j} \widetilde{s}_{l, j} \sum_{i \neq k} \widetilde{s}_{i, k}\right) \\
= & \frac{\sqrt{2}}{2} \mathbf{k}\left(\sum_{k \neq i} \widetilde{s}_{i, k} y_{j}+y_{j} \sum_{k \neq i} \widetilde{s}_{i, k}+y_{i} \sum_{l \neq j} \widetilde{s}_{l, j}+\sum_{l \neq j} \widetilde{s}_{l, j} y_{i}\right) \\
& +\frac{1}{2} \mathbf{k}^{2}\left(\sum_{l \neq j} \sum_{k \neq i} \widetilde{s}_{l, j} \widetilde{s}_{i, k}+\sum_{l \neq j} \sum_{k \neq i} \widetilde{s}_{i, k} \widetilde{s}_{l, j}\right)
\end{aligned}
$$

By Lemma 4.5 , the term $\frac{\sqrt{2}}{2}\left(\sum_{k \neq i} \widetilde{s}_{i, k} y_{j}+y_{j} \sum_{k \neq i} \widetilde{s}_{i, k}+y_{i} \sum_{l \neq j} \widetilde{s}_{l, j}+\sum_{l \neq j} \widetilde{s}_{l, j} y_{i}\right)$ is in $\operatorname{Seg}\left(W\left(\mathrm{~A}_{n-1}\right)\right)$. This completes the proof.

\section{Lemma 4.7.}

(1) $w x_{i}^{\prime} w^{-1}=x_{w(i)}^{\prime}$;

(2) $c_{i} x_{i}^{\prime}=-x_{i}^{\prime} c_{i}$ and $c_{j} x_{i}^{\prime}=x_{i}^{\prime} c_{j}$ for $i \neq j$. 
Proof. For (1), it suffices to show when $w=s_{\alpha}$ for some $\alpha \in \Delta$. Fix an $i$. By the definition of $x_{i}^{\prime}$, it suffices to show $s_{\alpha} y_{i}^{\prime} s_{\alpha}=y_{s_{\alpha}(i)}^{\prime}$. We consider two cases. In the case that $\left\langle e_{i}, \alpha\right\rangle=0, s_{\alpha}\left(\alpha_{i, j}\right)>0$ for any $j \neq i$. Then $s_{\alpha} \widetilde{s}_{i, j} s_{\alpha}=\widetilde{s}_{i, s_{\alpha}(j)}$ for any $j \neq i$. Thus, the last equality in Lemma 4.5 becomes

$$
s_{\alpha} y_{i}^{\prime} s_{\alpha}^{-1}=y_{i}+\frac{\sqrt{2}}{2} \mathbf{k} \sum_{j \neq i} \widetilde{s}_{i, s_{\alpha}(j)}=y_{i}^{\prime}
$$

In the case that $\left\langle e_{i}, \alpha\right\rangle \neq 0$, let $k=i-1$ or $i+1$ such that $\alpha=\alpha_{i, k}$. Then, by Lemmas 4.4(4) and 4.5,

$$
\begin{aligned}
s_{\alpha} y_{i}^{\prime} s_{\alpha}^{-1} & =y_{s_{\alpha}(i)}+\sqrt{2} \mathbf{k} \widetilde{s}_{\alpha}-\frac{\sqrt{2}}{2} \mathbf{k} \widetilde{s}_{\alpha}+\frac{\sqrt{2}}{2} \mathbf{k} \sum_{j \neq i, k} \widetilde{s}_{k, j} \\
& =y_{k}+\frac{\sqrt{2}}{2} \mathbf{k} \sum_{j \neq k} \widetilde{s}_{k, j} \\
& =y_{k}^{\prime} .
\end{aligned}
$$

For (2), it is straightforward from Lemma 4.4 and $y_{i}^{\prime}=x_{i}^{\prime} c_{i}$.

Remark 4.8. The subalgebra of $\mathbb{H}_{W\left(\mathrm{~A}_{n-1}\right)}^{\mathrm{Cl}}$ generated by the elements $y_{i}$ and $\widetilde{s}_{i, j}$ is the degenerate spin affine Hecke algebra of type $A_{n-1}$ defined in [Wan, Sect. 3.3]. (Other classical types for the degenerate spin affine Hecke algebra are established in [WK, Sect. 4].) The degenerate spin affine Hecke algebra can be regarded as a more elementary analogue of the degenerate affine Hecke algebra, and the notions of $y_{i}^{\prime}$ can be regarded as the Drinfield presentation [Dr] under the analogue.

Proposition 4.9. The degenerate affine Hecke-Clifford algebra $\mathbb{H}_{W\left(\mathrm{~A}_{n-1}\right)}^{\mathrm{Cl}}$ satisfies the property $(*)$ in Definition 3.1 .

Proof. We set $W$ in Definition 3.1 equal to $W\left(\mathrm{~A}_{n-1}\right)$ and set $a_{i}$ in Definition 3.1 to be $x_{i}^{\prime}$. Using Lemmas 4.6 and 4.7, one can verify relations (3.1) to (3.5) in Definition 3.1. By Proposition 4.3 and expressions of $x_{i}^{\prime},\left(x_{1}^{\prime}\right)^{m_{1}} \cdots\left(x_{n}^{\prime}\right)^{m_{n}} c_{1}^{\epsilon_{1}} \cdots c_{n}^{\epsilon_{n}} w$ $\left(m_{1}, \ldots, m_{n} \in \mathbb{Z}, \epsilon_{1}, \ldots, \epsilon_{n} \in\{0,1\}, w \in W\left(\mathrm{~A}_{n-1}\right)\right)$ form a basis for $\mathbb{H}_{W\left(\mathrm{~A}_{n-1}\right)}^{\mathrm{Cl}}$. These verify the property $(*)$.

\subsection{Type $\mathrm{B}_{n}$}

For type $\mathrm{B}_{n}$, we modify the original definition in [WK]. More precisely, the algebra we considered in Definition 4.11 is a deformation of the algebra in [WK]. It is not hard to do a similar modification for type $A_{n-1}$. The main reason for this modification is to construct an explicit module in the next section, which cannot be done in the original definition of [WK] (by our approach). Considering the lack of existing literature for the representation theory of the degenerate affine Hecke-Clifford algebra for other classical types, such examples may be interesting and important.

Notation 4.10. Let $W=W\left(\mathrm{~B}_{n}\right)$ be the Weyl group of type $\mathrm{B}_{n}$. Let the set $R\left(\mathrm{~B}_{n}\right)$ of roots for type $\mathrm{B}_{n}$ be

$$
R\left(\mathrm{~B}_{n}\right)=\left\{ \pm e_{i} \pm e_{j}: 1 \leq i<j \leq n\right\} \cup\left\{ \pm e_{i}: i=1, \ldots, n\right\} .
$$


The roots $\pm e_{i} \pm e_{j}(i \neq j)$ are long, while the roots $\pm e_{i}$ are short. Fix a set $R^{+}\left(\mathrm{B}_{n}\right)$ of positive roots:

$$
R^{+}\left(\mathrm{B}_{n}\right)=\left\{e_{i} \pm e_{j}: 1 \leq i<j \leq n\right\} \cup\left\{e_{i}: i=1, \ldots, n\right\} .
$$

The set $\Delta$ of simple roots is

$$
\left\{e_{i}-e_{i+1}: i=1, \ldots, n-1\right\} \cup\left\{e_{n}\right\} .
$$

For $i \neq j>0$, define $\alpha_{i j}$ as in (4.8), define $\alpha_{i,-j}=e_{i}+e_{j}$ and define $\alpha_{i}=e_{i}$. We also define $s_{i j}=s_{\alpha_{i, j}}, s_{i,-j}=s_{\alpha_{i,-j}}$ and $s_{i}=s_{\alpha_{i}}$.

We have a natural embedding $R\left(\mathrm{~A}_{n-1}\right) \subset R\left(\mathrm{~B}_{n}\right)$. and a natural embedding $W\left(\mathrm{~A}_{n-1}\right) \subset W\left(\mathrm{~B}_{n}\right)$ (i.e., the group $W\left(\mathrm{~A}_{n-1}\right)$ being the group generated by $s_{i, i+1}$ for $i=1, \ldots, n-1)$.

Definition 4.11. Let $N_{\mathrm{B}_{n}} \in \mathbb{C}$. Let $\mathbb{H}_{W\left(\mathrm{~B}_{n}\right)}^{\mathrm{Cl}}=\mathbb{H}_{W\left(\mathrm{~B}_{n}\right)}^{\mathrm{Cl}}\left(\mathbf{k}, N_{\mathrm{B}_{n}}\right)$ be the associative unital algebra generated by the symbols $\left\{x_{i}\right\}_{i=1}^{n},\left\{c_{i}\right\}_{i=1}^{n}$ and $\left\{f_{w}: w \in W\left(\mathrm{~B}_{n}\right)\right\}$ subject to the relations of (3), (4), (5), (6) in Definition 4.2 and additionally,

(1) the map from the group algebra $\mathbb{C}\left[W\left(\mathrm{~B}_{n}\right)\right]=\bigoplus_{w \in W\left(\mathrm{~B}_{n}\right)} \mathbb{C} w$ to $\mathbb{H}_{W\left(\mathrm{~B}_{n}\right)}^{\mathrm{Cl}}$ given by $w \mapsto f_{w}$ is an algebra injection;

(2) $f_{s_{n}} c_{n}=-c_{n} f_{s_{n}}$ and $f_{s_{n}} c_{i}=c_{i} f_{s_{n}}$ for $i \neq n$;

$$
\begin{aligned}
f_{s_{n}} x_{n}+x_{n} f_{s_{n}} & =-\sqrt{2} \mathbf{k}_{\alpha_{n}}, \\
f_{s_{n}} x_{j}-x_{j} f_{s_{n}} & =0 \quad \text { for } j \neq n ;
\end{aligned}
$$

(4) $x_{i} x_{j}-x_{j} x_{i}=N_{\mathrm{B}_{n}} c_{j} c_{i}$ for $i \neq j$.

We shall again simply write $w$ for $f_{w}$.

When $N_{\mathrm{B}_{n}}=0, \mathbb{H}_{W\left(\mathrm{~B}_{n}\right)}^{\mathrm{Cl}}\left(\mathbf{k}, N_{\mathrm{B}_{n}}\right)$ coincides with the degenerate affine HeckeClifford algebra of type $\mathrm{B}_{n}$ in [WK, Def. 3.9].

For $N_{\mathrm{B}_{n}} \neq 0$, while $x_{i}$ and $x_{j}$ does not commute for $i \neq j$, we still have $x_{i}^{2} x_{j}=x_{j} x_{i}^{2}$. The algebra $\mathbb{H}_{W\left(\mathrm{~B}_{n}\right)}^{\mathrm{Cl}}$ hence still has some nice properties such as the commutation relations with intertwining operators (but we do not need this in this paper).

For $i \neq j>0$, define $c_{\alpha_{i j}}$ as in (4.9) and define

$$
c_{\alpha_{i,-j}}=\frac{\sqrt{2}}{2}\left(c_{i}+c_{j}\right) .
$$

Set $\widetilde{s}_{i,-j}=s_{i,-j} c_{\alpha_{i,-j}}$. We also set $\widetilde{s}_{\alpha}=\widetilde{s}_{i}=s_{i} c_{i}$.

Since we have modified the original definition of the degenerate affine HeckeClifford algebra for type $\mathrm{B}_{n}$ in $[\mathrm{WK}]$, we will give a proof for the existence of the PBW type basis.

Proposition 4.12. The set

$$
\left\{x_{1}^{m_{1}} \cdots x_{n}^{m_{n}} c_{1}^{\epsilon_{1}} \cdots c_{n}^{\epsilon_{n}} w: m_{1}, \ldots, m_{n} \in \mathbb{Z}_{\geq 0}, \epsilon_{1}, \ldots, \epsilon_{n} \in\{0,1\}, w \in W\left(\mathrm{~B}_{n}\right)\right\}
$$

forms a basis for $\mathbb{H}_{W\left(\mathrm{~B}_{n}\right)}^{\mathrm{Cl}}$. 
Proof. We follow the argument in [Kl, Thm. 3.2.2]. We consider the algebra $\widetilde{\mathbb{H}}$ generated by $\left\{\bar{x}_{i}\right\},\left\{\bar{c}_{i}\right\}$ and $\left\{\bar{s}_{i, i+1}\right\}_{i=1}^{n-1} \cup\left\{\bar{s}_{n}\right\}$ subject to the relations (3), (4), (5), (6) in Definition 4.2 and the relation (2) (but not (1)) in Definition 4.18 (with a trivial replacement of notations). We resolve the minimal ambiguities according to the Bergman's diamond lemma [Be]. For example, we may consider an ordering $\bar{s}<\bar{c}_{n}<\cdots<\bar{c}_{1}<\bar{x}_{n}<\cdots<\bar{x}_{1}$, where $s$ is any simple reflection in $W\left(\mathrm{~B}_{n}\right)$. This induces a semigroup ordering on $\left\langle\bar{x}_{i}, \bar{c}_{i}, \bar{s}\right\rangle(i=1, \ldots, n$ and $s$ runs for all simple reflections) from the length of words and the lexicographical ordering. Then one checks that

$$
\begin{aligned}
\left(\bar{s}_{i, i+1} \bar{x}_{i+1}\right) \bar{x}_{i} & =\left(\bar{x}_{i} \bar{s}_{i, i+1}-\mathbf{k}_{\alpha_{i, i+1}}\left(-1+\bar{c}_{i+1} \bar{c}_{i}\right)\right) \bar{x}_{i} \\
& =\bar{x}_{i} \bar{s}_{i, i+1} \bar{x}_{i}-\mathbf{k}_{\alpha_{i, i+1}}\left(-1+\bar{c}_{i+1} \bar{c}_{i}\right) \bar{x}_{i} \\
& =\bar{x}_{i} \bar{x}_{i+1} \bar{s}_{i, i+1}+\mathbf{k}_{\alpha_{i, i+1}} \bar{x}_{i}\left(-1+\bar{c}_{i} \bar{c}_{i+1}\right)-\mathbf{k}_{\alpha_{i, i+1}}\left(-1+\bar{c}_{i+1} \bar{c}_{i}\right) \bar{x}_{i} \\
& =\bar{x}_{i} \bar{x}_{i+1} \bar{s}_{i, i+1}
\end{aligned}
$$

and

$$
\begin{aligned}
\bar{s}_{i, i+1}\left(\bar{x}_{i+1} \bar{x}_{i}\right)= & \bar{s}_{i, i+1}\left(\bar{x}_{i} \bar{x}_{i+1}+N_{\mathrm{B}_{n}} \bar{c}_{i} \bar{c}_{i+1}\right) \\
= & \bar{s}_{i, i+1} \bar{x}_{i} \bar{x}_{i+1}+N_{\mathrm{B}_{n}} \bar{c}_{i+1} \bar{c}_{i} \bar{s}_{i, i+1} \\
= & \bar{x}_{i+1} \bar{s}_{i, i+1} \bar{x}_{i+1}+\mathbf{k}_{\alpha_{i, i+1}}\left(-1+\bar{c}_{i} \bar{c}_{i+1}\right) x_{i+1}+N_{\mathrm{B}_{n}} \bar{c}_{i+1} \bar{c}_{i} \bar{s}_{i, i+1} \\
= & \bar{x}_{i+1} \bar{x}_{i} \bar{s}_{i, i+1}-\mathbf{k}_{\alpha_{i, i+1}} \bar{x}_{i+1}\left(-1+\bar{c}_{i+1} \bar{c}_{i}\right) \\
& +\mathbf{k}_{\alpha_{i, i+1}}\left(-1+\bar{c}_{i} \bar{c}_{i+1}\right) \bar{x}_{i+1}+N_{\mathrm{B}_{n}} \bar{c}_{i+1} \bar{c}_{i} \bar{s}_{i, i+1} \\
= & \bar{x}_{i} \bar{x}_{i+1} \bar{s}_{i, i+1} .
\end{aligned}
$$

Similarly,

$$
\begin{aligned}
\left(\bar{s}_{n} \bar{x}_{n}\right) \bar{x}_{j} & =\left(-\bar{x}_{n} \bar{s}_{n}-\sqrt{2} \mathbf{k}_{\alpha_{n}}\right) \bar{x}_{j} \\
& =-\bar{x}_{n} \bar{s}_{n} \bar{x}_{j}-\sqrt{2} \mathbf{k}_{\alpha_{n}} \bar{x}_{j} \\
& =-\bar{x}_{n} \bar{x}_{j} \bar{s}_{n}-\sqrt{2} \mathbf{k}_{\alpha_{n}} \bar{x}_{j} \\
& =-\bar{x}_{j} \bar{x}_{n} \bar{s}_{n}-N_{\mathrm{B}_{n}} c_{j} c_{n} \bar{s}_{n}-\sqrt{2} \mathbf{k}_{\alpha_{n}} \bar{x}_{j}
\end{aligned}
$$

and

$$
\begin{aligned}
\bar{s}_{n}\left(\bar{x}_{n} \bar{x}_{j}\right) & =\bar{s}_{n} \bar{x}_{j} \bar{x}_{n}+N_{\mathrm{B}_{n}} \bar{s}_{n} \bar{c}_{j} \bar{c}_{n} \\
& =\bar{x}_{j} \bar{s}_{n} \bar{x}_{n}+N_{\mathrm{B}_{n}} \bar{s}_{n} \bar{c}_{j} \bar{c}_{n} \\
& =-\bar{x}_{j} \bar{x}_{n} \bar{s}_{n}-\sqrt{2} \mathbf{k}_{\alpha_{n}} \bar{x}_{j}-N_{\mathrm{B}_{n}} \bar{c}_{j} \bar{c}_{n} \bar{s}_{n} .
\end{aligned}
$$

Similarly, for $i>j>k$,

$$
\begin{aligned}
\left(\bar{x}_{i} \bar{x}_{j}\right) \bar{x}_{k} & =\bar{x}_{j} \bar{x}_{i} \bar{x}_{k}+N_{\mathrm{B}_{n}} c_{j} c_{i} \bar{x}_{k} \\
& =\bar{x}_{j} \bar{x}_{k} \bar{x}_{i}+N_{\mathrm{B}_{n}} \bar{x}_{j} c_{k} c_{i}+N_{\mathrm{B}_{n}} c_{j} c_{i} \bar{x}_{k} \\
& =\bar{x}_{k} \bar{x}_{j} \bar{x}_{i}+N_{\mathrm{B}_{n}} c_{k} c_{j} \bar{x}_{i}+N_{\mathrm{B}_{n}} \bar{x}_{j} c_{k} c_{i}+N_{\mathrm{B}_{n}} c_{j} c_{i} \bar{x}_{k} \\
& =\bar{x}_{k} \bar{x}_{j} \bar{x}_{i}+N_{\mathrm{B}_{n}} c_{k} c_{j} \bar{x}_{i}+N_{\mathrm{B}_{n}} c_{k} c_{i} \bar{x}_{j}+N_{\mathrm{B}_{n}} c_{j} c_{i} \bar{x}_{k} .
\end{aligned}
$$


The calculation for $\bar{x}_{i}\left(\bar{x}_{j} \bar{x}_{k}\right)$ is similar. Other minimal ambiguities can be checked similarly.

Let $\mathcal{I}$ be the two-sided ideal of $\widetilde{\mathbb{H}}$ generated by the relations of $W\left(\mathrm{~B}_{n}\right)$ (e.g., $\left.\bar{s}^{2}-1, \bar{s}_{i, i+1} \bar{s}_{i+1, i+2} \bar{s}_{i, i+1}-\bar{s}_{i+1, i+2} \bar{s}_{i, i+1} \bar{s}_{i+1, i+2}\right)$. Then $\widetilde{H} / \mathcal{I} \cong \mathbb{H}_{W\left(\mathrm{~B}_{n}\right)}^{\mathrm{Cl}}$. Let $\mathcal{P}$ be the subalgebra of $\widetilde{\mathbb{H}}$ generated by by $x_{i}$ and $c_{i}$. It is straightforward to check that $\left(\bar{s}^{2}-1\right) \mathcal{P}=\mathcal{P}\left(\bar{s}^{2}-1\right),\left(\bar{s}_{i, i+1} \bar{s}_{i+1, i+2} \bar{s}_{i, i+1}-\bar{s}_{i+1, i+2} \bar{s}_{i, i+1} \bar{s}_{i+1, i+2}\right) \mathcal{P}=$ $\mathcal{P}\left(\bar{s}_{i, i+1} \bar{s}_{i+1, i+2} \bar{s}_{i, i+1}-\bar{s}_{i+1, i+2} \bar{s}_{i, i+1} \bar{s}_{i+1, i+2}\right)$, and other similar equations. Those equations can also be deduced from Lemma 4.14 and its proof below.

Lemma 4.13. For any root $\alpha>0, c_{i} \widetilde{s}_{\alpha}=-\widetilde{s}_{\alpha} c_{i}$.

\section{Lemma 4.14.}

$$
w y_{i} w^{-1}=y_{w(i)}+\sqrt{2} \sum_{\alpha>0, w^{-1}(\beta)<0,\left\langle\beta, w\left(e_{i}\right)\right\rangle \neq 0} \mathbf{k}_{\alpha} \widetilde{s}_{\alpha} .
$$

In particular, for $\alpha>0$

$$
\begin{aligned}
\widetilde{s}_{\alpha} y_{i} \widetilde{s}_{\alpha}^{-1}+y_{s_{\alpha}(i)} & =-\sqrt{2} \sum_{\alpha>0, s_{\alpha}^{-1}(\beta)<0,\left\langle\beta, s_{\alpha}\left(e_{i}\right)\right\rangle \neq 0} \mathbf{k}_{\beta} \widetilde{s}_{\beta} .
\end{aligned}
$$

Proof. The relation $s_{n} x_{n}+x_{n} s_{n}=-\sqrt{2} \mathbf{k}_{\alpha}$ implies $s_{n} y_{n}-y_{n} s_{n}=-\sqrt{2} \mathbf{k}_{\alpha} c_{n}$. The latter equation is also equivalent to $s_{n} y_{n} s_{n}^{-1}=y_{n}+\sqrt{2} \mathbf{k}_{\alpha} \widetilde{s}_{n}$. The remaining proof is just similar to the case of $\mathrm{A}_{n-1}$ in the proof of Lemma 4.5.

For $i>0$, define $y_{i}=x_{i} c_{i}$.

$$
y_{i}^{\prime}=y_{i}+\frac{\sqrt{2}}{2} \sum_{\alpha>0,\left\langle\alpha, e_{i}\right\rangle \neq 0} \mathbf{k}_{\alpha} \widetilde{s}_{\alpha} .
$$

We also define $y_{-i}=y_{i}$ and $y_{-i}^{\prime}=y_{i}^{\prime}$.

There is a natural permutation of $W\left(\mathrm{~B}_{n}\right)$ on the set $\{ \pm 1, \ldots, \pm n\}$.

\section{Lemma 4.15.}

(1) For any $w \in W\left(\mathrm{~B}_{n}\right), w y_{i}^{\prime} w^{-1}=y_{w(i)}^{\prime}$.

(2) For $i \neq j, y_{i}^{\prime} y_{j}^{\prime}+y_{j}^{\prime} y_{i}^{\prime} \in \operatorname{Seg}\left(W\left(\mathrm{~B}_{n}\right)\right)$.

Proof. For (1), it suffices to check when $w=s_{\alpha}$ is a simple reflection. It is the direct consequence of the expression (4.10) for $y_{i}^{\prime}$, Lemma 4.14, and the fact that $s_{\alpha} \widetilde{s}_{\alpha} s_{\alpha}=-\widetilde{s}_{\alpha}$. For (2), using the expression (4.10), we have

$$
\begin{aligned}
& y_{i}^{\prime} y_{j}^{\prime}+y_{j}^{\prime} y_{i}^{\prime}=y_{i} y_{j}+y_{j} y_{i}+\frac{\sqrt{2}}{2}\left[\sum_{\alpha>0,\left\langle\alpha, e_{j}\right\rangle \neq 0} \mathbf{k}_{\alpha}\left(y_{i} \widetilde{s}_{\alpha}+\widetilde{s}_{\alpha} y_{i}\right)+\sum_{\alpha>0,\left\langle\alpha, e_{i}\right\rangle \neq 0} \mathbf{k}_{\alpha}\left(y_{j} \widetilde{s}_{\alpha}+\widetilde{s}_{\alpha} y_{j}\right)\right] \\
& +\frac{1}{2} \sum \mathbf{k}_{\alpha} \mathbf{k}_{\beta}\left(\widetilde{s}_{\alpha} \widetilde{s}_{\beta}+\widetilde{s}_{\beta} \widetilde{s}_{\alpha}\right) \text {. } \\
& \alpha, \beta>0,\left\langle\alpha, e_{i}\right\rangle \neq 0,\left\langle\beta, e_{i}\right\rangle \neq 0
\end{aligned}
$$


Since $y_{i} y_{j}+y_{j} y_{i}=N_{\mathrm{B}_{n}}$, we only need to consider and show that the middle term is in $\operatorname{Seg}\left(W\left(\mathrm{~B}_{n}\right)\right)$ :

$$
\begin{aligned}
\sum_{\alpha>0,\left\langle\alpha, e_{j}\right\rangle \neq 0}\left(y_{i} \widetilde{s}_{\alpha}+\widetilde{s}_{\alpha} y_{i}\right)+\sum_{\alpha>0,\left\langle\alpha, e_{i}\right\rangle \neq 0}\left(y_{j} \widetilde{s}_{\alpha}+\widetilde{s}_{\alpha} y_{j}\right) \\
=\left(\sum_{k \neq i} \mathbf{k}_{\alpha_{i, k}} \widetilde{s}_{i, k} y_{j}+y_{j} \sum_{k \neq i} \mathbf{k}_{\alpha_{i, k}} \widetilde{s}_{i, k}+y_{i} \sum_{l \neq j} \mathbf{k}_{\alpha_{l, j}} \widetilde{s}_{l, j}+\sum_{l \neq j} \mathbf{k}_{\alpha_{l, j}} \widetilde{s}_{l, j} y_{i}\right) \\
\quad+\left(\sum_{k>0, k \neq i} \widetilde{s}_{i,-k} y_{j}+y_{j} \sum_{k>0, k \neq i} \widetilde{s}_{i,-k}+y_{i} \sum_{l>0, l \neq j} \widetilde{s}_{j,-l}+\sum_{l>0, l \neq j} \widetilde{s}_{j,-l} y_{i}\right) \\
\quad+\mathbf{k}_{\alpha_{i}}\left(\widetilde{s}_{i} y_{j}+y_{j} \widetilde{s}_{i}\right)+\mathbf{k}_{\alpha_{j}}\left(\widetilde{s}_{j} y_{i}+y_{i} \widetilde{s}_{i}\right)
\end{aligned}
$$

which is in $\operatorname{Seg}\left(W\left(\mathrm{~B}_{n}\right)\right)$ by Lemma 4.14 .

Proposition 4.16. The superalgebra $\mathbb{H}_{W\left(\mathrm{~B}_{n}\right)}^{\mathrm{Cl}}$ satisfies the property $(*)$.

Proof. Let $x_{i}^{\prime}=-y_{i}^{\prime} c_{i}$. We set $W$ in Definition 3.1 to be $W\left(\mathrm{~B}_{n}\right)$ and $a_{i}$ to be $x_{i}^{\prime}$. With Lemma 4.15, one can verify relations (3.1) to (3.5) in Definition 3.1 (also see more detail for type $\mathrm{A}_{n-1}$ in Section 4.1). By Proposition 4.12, $\mathbb{H}_{W\left(\mathrm{~B}_{n}\right)}^{\mathrm{Cl}}$ has a PBW type basis. These show the proposition.

\subsection{Type $\mathrm{D}_{n}$}

Notation 4.17. Let $W\left(D_{n}\right)$ be the Weyl group of type $D_{n}$. Let the set $R\left(D_{n}\right)$ of roots for type $D_{n}$ be

$$
R\left(\mathrm{D}_{n}\right)=\left\{ \pm e_{i} \pm e_{j}: 1 \leq i<j \leq n\right\} \subset R\left(\mathrm{~B}_{n}\right) .
$$

Let $R^{+}\left(\mathrm{D}_{n}\right)=R\left(\mathrm{D}_{n}\right) \cap R^{+}\left(\mathrm{B}_{n}\right)$ be a fixed set of positive roots. We shall again write $\alpha>0$ for $\alpha \in R^{+}\left(\mathrm{D}_{n}\right)$ and $\alpha<0$ for $-\alpha \in R\left(\mathrm{D}_{n}\right)$. The set of simple roots is given by

$$
\Delta=\left\{e_{i}-e_{i+1}: i=1, \ldots, n-1\right\} \cup\left\{e_{n-1}+e_{n}\right\} .
$$

Since there is only one $W$-orbit for $R\left(\mathrm{D}_{n}\right)$, we simply write $\mathbf{k}$ for $\mathbf{k}_{\alpha}$ for any $\alpha \in R\left(\mathrm{D}_{n}\right)$.

We shall regard $W\left(\mathrm{D}_{n}\right)$ as the subgroup of $W\left(\mathrm{~B}_{n}\right)$ generated by elements $s_{i, j}$ and $s_{i,-j}$ for $i, j>0$. We shall also keep using the notations in Notation 4.10.

Definition 4.18. Let $N_{\mathrm{D}_{n}} \in \mathbb{C}$. Let $\mathbf{k}^{B}: R\left(\mathrm{~B}_{n}\right) \rightarrow \mathbb{C}$ such that $\left.\mathbf{k}^{B}\right|_{R\left(\mathrm{D}_{n}\right)}=\mathbf{k}$ and $\mathbf{k}_{\alpha}^{B}=0$ for any short root $\alpha$ in $R\left(\mathrm{~B}_{n}\right)$. Let $\mathbb{H}_{W\left(\mathrm{D}_{n}\right)}^{\mathrm{Cl}}=\mathbb{H}_{W\left(\mathrm{D}_{n}\right)}^{\mathrm{Cl}}\left(\mathbf{k}, N_{\mathrm{D}_{n}}\right)$ be the supersubalgebra of $\mathbb{H}_{W\left(\mathrm{~B}_{n}\right)}^{\mathrm{Cl}}\left(\mathbf{k}^{B}, N_{\mathrm{D}_{n}}\right)$ generated by the elements $w \in W\left(\mathrm{D}_{n}\right) \subset$ $W\left(\mathrm{~B}_{n}\right),\left\{x_{i}\right\}_{i=1}^{n}$ and $\left\{c_{i}\right\}_{i=1}^{n}$.

Remark 4.19. We can explicitly write down the commutation formula from the algebra structure of $\mathbb{H}_{W\left(\mathrm{~B}_{n}\right)}^{\mathrm{Cl}}$. For example,

$$
\begin{aligned}
s_{n-1,-n} x_{n-1}+x_{n} s_{n-1,-n} & =s_{n} s_{n-1, n} s_{n} x_{n-1}+x_{n} s_{n} s_{n-1, n} s_{n} \\
& =-s_{n} s_{n-1, n} x_{n} s_{n}+s_{n} x_{n-1} s_{n-1, n} s_{n} \\
& =s_{n}\left(-s_{n-1, n} x_{n}+x_{n-1} s_{n-1, n}\right) s_{n} \\
& =s_{n}\left(\mathbf{k}\left(-1+c_{n} c_{n-1}\right)\right) s_{n} \\
& =\mathbf{k}\left(-1+c_{n-1} c_{n}\right) .
\end{aligned}
$$


This agrees with a relation in [WK, Def. 3.6]. When $N_{\mathrm{D}_{n}}=0, \mathbb{H}_{W\left(\mathrm{D}_{n}\right)}^{\mathrm{Cl}}(\mathbf{k}, 0)$ is isomorphic to the degenerate affine Hecke-Clifford algebra of type $\mathrm{D}_{n}$ defined in [WK, Def. 3.6]. (We remark that in [WK], their convention for $c_{i}$ satisfies $c_{i}^{2}=1$ rather than $c_{i}^{2}=-1$.)

We again define

$$
y_{i}^{\prime}=y_{i}+\frac{\sqrt{2}}{2} \mathbf{k} \sum_{\alpha>0,\left\langle\alpha, e_{i}\right\rangle \neq 0} \widetilde{s}_{\alpha}=y_{i}+\frac{\sqrt{2}}{2} \mathbf{k} \sum_{j \neq i} \widetilde{s}_{i j}+\frac{\sqrt{2}}{2} \mathbf{k} \sum_{j \neq i} \widetilde{s}_{i,-j} .
$$

Again, for notational convenience, set $y_{-i}^{\prime}=y_{i}^{\prime}$.

\section{Lemma 4.20.}

(1) $c_{i} y_{j}^{\prime}=-y_{j}^{\prime} c_{i}$ for any $i, j$;

(2) $s_{\alpha} y_{i}^{\prime} s_{\alpha}^{-1}=y_{s_{\alpha}(i)}^{\prime}$;

(3) for $i \neq j, y_{i}^{\prime} y_{j}^{\prime}+y_{j}^{\prime} y_{i}^{\prime} \in \operatorname{Seg}\left(W\left(\mathrm{D}_{n}\right)\right)$.

Proof. Note that $y_{i}^{\prime}$ is defined as the one in (4.10) for type $\mathrm{B}_{n}$ in Section 4.2 since we have $\mathbf{k}_{\alpha}^{B}=0$ for any short root $\alpha \in R\left(\mathrm{~B}_{n}\right)$. Then the results can be established by Lemma 4.14 and by investigating the proof of Lemma 4.15 .

Proposition 4.21. The algebra $\mathbb{H}_{W\left(\mathrm{D}_{n}\right)}^{\mathrm{Cl}}$ satisfies the property $(*)$ in Definition 3.1.

Proof. This follows from $\mathbb{H}_{W\left(\mathrm{D}_{n}\right)}^{C l}$ forming a supersubalgebra of $\mathbb{H}_{W\left(\mathrm{~B}_{n}\right)}^{\mathrm{Cl}}\left(\mathbf{k}^{B}, N_{\mathrm{D}_{n}}\right)$ and Remark 4.19.

\subsection{Dirac element $D$}

Let $\mathbb{H}=\mathbb{H}_{W\left(\mathrm{~A}_{n-1}\right)}^{\mathrm{Cl}}, \mathbb{H}_{W\left(\mathrm{~B}_{n}\right)}^{\mathrm{Cl}}$, or $\mathbb{H}_{W\left(\mathrm{D}_{n}\right)}^{\mathrm{Cl}}$. Using (3.6), the Dirac element $D$ for $\mathbb{H}$ is defined as

$$
D=\sum_{i=1}^{n} x_{i}^{\prime} c_{i}
$$

Using the expressions in Section 4.1, the explicit form of the Dirac element $D$ is as:

(1) Type $\mathrm{A}_{n-1}$ and $\mathrm{D}_{n}$ :

$$
D=\sum_{i=1}^{n} x_{i} c_{i}+\sqrt{2} \sum_{\alpha>0} \mathbf{k}_{\alpha} s_{\alpha} c_{\alpha}=\sum_{i=1}^{n} y_{i}+\sqrt{2} \sum_{\alpha>0} \mathbf{k}_{\alpha} \widetilde{s}_{\alpha}
$$

(2) Type $\mathrm{B}_{n}$ :

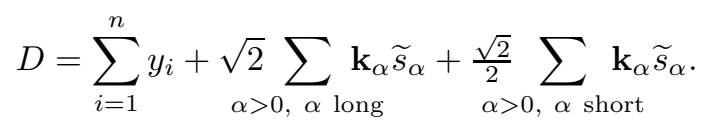

In types $\mathrm{A}_{n-1}$ and $\mathrm{D}_{n}$, we consider that all the roots are long. 


\section{Lemma 4.22.}

$$
\left(\sum_{\alpha>0, \alpha \text { long }} \widetilde{s}_{\alpha}\right)^{2}=\sum_{\substack{\alpha>0, \beta>0, s_{\alpha}(\beta)<0 \\ \alpha, \beta \text { long }}} \widetilde{s}_{\alpha} \widetilde{s}_{\beta} .
$$

The above equality is also true if we replace all the long roots by short roots. Similarly, we also have

$$
\left(\sum_{\alpha>0, \alpha \text { long }} \widetilde{s}_{\alpha}\right)\left(\sum_{\alpha>0, \alpha \text { short }} \widetilde{s}_{\alpha}\right)+\left(\sum_{\alpha>0, \alpha \text { short }} \widetilde{s}_{\alpha}\right)\left(\sum_{\alpha>0, \alpha \text { long }} \widetilde{s}_{\alpha}\right)=\sum_{\alpha>0, \beta>0, s_{\alpha}(\beta)<0} \widetilde{s}_{\alpha} \widetilde{s}_{\beta},
$$

where $\alpha$ and $\beta$ run for all pairs of roots with distinct length.

Proof. We only prove for the first case, that is, the case of long roots only. It suffices to show that

$$
\sum_{\substack{\alpha>0, \beta>0, s_{\alpha}(\beta)>0 \\ \alpha, \beta \text { long }}} \widetilde{s}_{\alpha} \widetilde{s}_{\beta}=0 .
$$

Set $\widetilde{R}=\left\{(\alpha, \beta) \in R^{+} \times R^{+}: s_{\alpha}(\beta)>0, \alpha\right.$ and $\beta$ are long $\}$. Note that for any $(\alpha, \beta) \in \widetilde{R}$, either $s_{\beta}(\alpha)>0$ or $s_{s_{\alpha}(\beta)}(\alpha)>0$. We define a map $\iota: \widetilde{R} \rightarrow \widetilde{R}$ such that

$$
\iota(\alpha, \beta)= \begin{cases}\left(\beta, s_{\beta}(\alpha)\right) & \text { if } s_{\beta}(\alpha)>0 \\ \left(s_{\alpha}(\beta), \alpha\right) & \text { if } s_{s_{\alpha}(\beta)}(\alpha)>0 .\end{cases}
$$

It is not hard to verify that $\iota$ is well-defined and is an involution. For $\iota(\alpha, \beta)=$ $\left(\alpha^{\prime}, \beta^{\prime}\right)$, one can also check that $\widetilde{s}_{\alpha} \widetilde{s}_{\beta}+\widetilde{s}_{\alpha^{\prime}} \widetilde{s}_{\beta^{\prime}}=0$. Thus each term $\widetilde{s}_{\alpha} \widetilde{s}_{\beta}$ in the expression $\sum_{\alpha>0, \beta>0, s_{\alpha}(\beta)>0} \widetilde{s}_{\alpha} \widetilde{s}_{\beta}$ can be paired with another one and get canceled. This proves the expression is zero.

By Proposition 4.9, Proposition 4.16, and Proposition 4.21, $\mathbb{H}$ satisfies the property $(*)$ and hence we can define $\operatorname{Seg}(W)$ to be a subalgebra of $\mathbb{H}$ according to Definition 3.1.

We compute the square of the Dirac element $D$. This is an analogue of [BCT, Thm. 2.11].

Theorem 4.23. Let $\mathbb{H}=\mathbb{H}_{W\left(\mathrm{~A}_{n-1}\right)}^{\mathrm{Cl}}, \mathbb{H}_{W\left(\mathrm{~B}_{n}\right)}^{\mathrm{Cl}}$, or $\mathbb{H}_{W\left(\mathrm{D}_{n}\right)}^{\mathrm{Cl}}$. Then

$$
D^{2}=\Omega_{\mathbb{H}}-\Omega_{\mathrm{Seg}(W)},
$$

where

$$
\begin{aligned}
\Omega_{\mathbb{H}} & =\sum_{i=1}^{n} x_{i}^{2}, \\
\Omega_{\operatorname{Seg}(W)} & =\frac{1}{2} \sum_{\alpha>0, \beta>0, s_{\alpha}(\beta)<0}|\langle\alpha, \alpha\rangle||\langle\beta, \beta\rangle| \mathbf{k}_{\alpha} \mathbf{k}_{\beta} \widetilde{s}_{\alpha} \widetilde{s}_{\beta} .
\end{aligned}
$$

Moreover, $\mathbb{H}$ satisfies the property $(* *)$. 
Proof. We only do for types $\mathrm{A}_{n-1}$ and $\mathrm{B}_{n}$, and the case for type $\mathrm{D}_{n}$ follows from type $\mathrm{B}_{n}$.

By Lemma 4.5 and Lemma 4.14, for any $\alpha>0$,

$$
\mathbf{k}_{\alpha}\left(\sum_{i=1}^{n} y_{i} \widetilde{s}_{\alpha}+\widetilde{s}_{\alpha} \sum_{i=1}^{n} y_{i}\right)=-\sqrt{2} \mathbf{k}_{\alpha} \sum_{\beta>0, s_{\alpha}(\beta)<0} \mathbf{k}_{\beta}|\langle\beta, \beta\rangle| \widetilde{s}_{\alpha} \widetilde{s}_{\beta} .
$$

Now, by (4.13) and Lemma 4.22,

$$
\begin{aligned}
& D^{2}=\left(\sum_{i=1}^{n} y_{i}+\frac{\sqrt{2}}{2} \sum_{\alpha>0} \mathbf{k}_{\alpha}|\langle\alpha, \alpha\rangle| \widetilde{s}_{\alpha}\right)^{2} \\
& =\left(\sum_{i=1}^{n} y_{i}\right)^{2}+\frac{\sqrt{2}}{2} \sum_{i=1}^{n} y_{i} \sum_{\alpha>0} \mathbf{k}_{\alpha}|\langle\alpha, \alpha\rangle| \widetilde{s}_{\alpha}+\frac{\sqrt{2}}{2} \sum_{\alpha>0} \mathbf{k}_{\alpha}|\langle\alpha, \alpha\rangle| \widetilde{s}_{\alpha} \sum_{i=1}^{n} y_{i} \\
& +\frac{1}{2}\left(\sum_{\alpha>0} \mathbf{k}_{\alpha}|\langle\alpha, \alpha\rangle| \widetilde{s}_{\alpha}\right)^{2} \\
& =\sum_{i=1}^{n} x_{i}^{2}-\frac{1}{2} \sum_{\alpha>0, \beta>0, s_{\alpha}(\beta)<0} \mathbf{k}_{\alpha} \mathbf{k}_{\beta}|\langle\alpha, \alpha\rangle \|\langle\beta, \beta\rangle| \widetilde{s}_{\alpha} \widetilde{s}_{\beta} .
\end{aligned}
$$

We can directly verify that $\Omega_{\mathbb{H}}$ is in the center of $\mathbb{H}$ and $\Omega_{\operatorname{Seg}(W)}$ is in the center of $\operatorname{Seg}(W)$. Hence, $\mathbb{H}$ has the property $(* *)$.

We obtain the following Parthasarathy-Dirac-type inequality. Examples satisfying the hypothesis of Corollary 4.24 below will be considered in Section 7 (see Proposition 7.12).

Corollary 4.24. Suppose an irreducible $\mathbb{H}$-module $(\pi, X)$ satisfies the property that $X$ admits a non-degenerate positive-definite Hermitian form such that the adjoint operator of $\pi(D)$ is $-\pi(D)$. For any irreducible $\operatorname{Seg}(W)$-module $(\sigma, U)$,

$$
\operatorname{Hom}_{\operatorname{Seg}(W)}\left(U, \operatorname{Res}_{\operatorname{Seg}(W)}^{\mathbb{H}} X\right) \neq 0
$$

only if

$$
\chi_{\pi}\left(\Omega_{\mathbb{H}}\right) \leq \chi_{\sigma}\left(\Omega_{\operatorname{Seg}(W)}\right) .
$$

Proof. Let $U_{X}$ be an $U$-isotypical component of $X$ and let $u \in U$. The corollary follows from

$$
0 \leq\langle D . u, D . u\rangle=\left\langle u,-D^{2} . u\right\rangle=-\left(\chi_{\pi}\left(\Omega_{\mathbb{H}}\right)-\chi_{\sigma}\left(\Omega_{\operatorname{Seg}(W)}\right)\right)\langle u, u\rangle .
$$

The conclusion of this section is a version of Theorem 3.5 in specific cases.

Theorem 4.25. Let $\mathbb{H}=\mathbb{H}_{W\left(\mathrm{~A}_{n-1}\right)}^{\mathrm{Cl}}, \mathbb{H}_{W\left(\mathrm{~B}_{n}\right)}^{\mathrm{Cl}}$, or $\mathbb{H}_{W\left(\mathrm{D}_{n}\right)}^{\mathrm{Cl}}$. Let $(\pi, X)$ be an irreducible supermodule of $\mathbb{H}$ with the central character $\chi_{\pi}$ (Definition 2.6). Let $D$ be the Dirac element in $\mathbb{H}$ in (4.12). Define the Dirac cohomology $H_{D}(X)$ as in Theorem 3.5. Then $H_{D}(X)$ has a natural $\operatorname{Seg}(W)$-module structure. Suppose

$$
\operatorname{Hom}_{\operatorname{Seg}(W)}\left(U, H_{D}(X)\right) \neq 0,
$$

for some $\operatorname{Seg}(W)$-module $(\sigma, U)$. Then $\chi_{\pi}=\chi^{\sigma}$, where $\chi^{\sigma}$ is defined as in (3.7).

Proof. This immediately follows from Theorem 3.5, Proposition 4.9, and Theorem 4.23 . 


\section{Examples of non-vanishing Dirac cohomology}

\subsection{Construction of some modules}

In this section, we construct some modules for the degenerate affine Hecke-Clifford algebra of classical types.

In type $A_{n-1}$, we follow the construction in [HKS, Sect. 4.1], which uses a Jucys-Murphy-type element. For type $\mathrm{B}_{n}$, we use a slightly different approach. The underlying idea of the construction is to first consider a $\operatorname{Seg}(W)$-module and then try to extend the action to the entire degenerate affine Hecke-Clifford algebra. However, we may not expect that this process always works, and indeed, we can only do it for certain parameters.

Type $\mathrm{A}_{n-1}$ : Let $\mathrm{Cl}_{n}$ be the subalgebra of $\mathbb{H}_{W\left(\mathrm{~A}_{n-1}\right)}^{\mathrm{Cl}}$ generated by all $c_{i}$. Define $\widetilde{\mathrm{St}} W\left(\mathrm{~A}_{n-1}\right)$ to be an $\mathbb{H}_{W\left(\mathrm{~A}_{n-1}\right)}^{\mathrm{Cl}}$-supermodule, which is identified with $\mathrm{Cl}_{n}$ as vector spaces and the action of $\mathbb{H}^{n}$ on $\widetilde{\mathrm{St}}_{W\left(\mathrm{~A}_{n-1}\right)}$ is determined by the following:

$$
\begin{gathered}
c_{i} \cdot 1=c_{i}, \\
s_{\alpha} \cdot 1=1,
\end{gathered}
$$

where 1 is the identity in $\mathrm{Cl}_{n}$ and

$$
x_{i} . v=\mathbf{k}\left(\sum_{1 \leq j<i \leq n} s_{i, j}\left(1-c_{i} c_{j}\right)\right) \cdot v,
$$

where $v$ is any vector in $\mathrm{Cl}_{n}$ and the actions of $s_{i, j}$ and $c_{i}, c_{j}$ are the ones defined in (5.14) and (5.15). The notation $\widetilde{\mathrm{St}}_{W\left(\mathrm{~A}_{n-1}\right)}$ stands for a Steinberg-type module as it performs the role of Steinberg module in the degenerate affine Hecke algebra. It is straightforward to check that the above actions define an $\mathbb{H}_{W\left(\mathrm{~A}_{n-1}\right)}^{\mathrm{Cl}}$-module by verifying the defining relations of $\mathbb{H}_{W\left(\mathrm{~A}_{n-1}\right)}^{\mathrm{Cl}}$. Some details can be found in $[\mathrm{HKS}$, Prop. 4.1.1].

Type $\mathrm{B}_{n}$ : Let $\alpha$ be a long root in $R\left(\mathrm{~B}_{n}\right)$ and let $\beta$ be a short root in $R\left(\mathrm{~B}_{n}\right)$. Set $N_{\mathrm{B}_{n}}=2(n-1) \mathbf{k}_{\alpha}^{2}+\sqrt{2} \mathbf{k}_{\alpha} \mathbf{k}_{\beta}$. Let $\mathrm{Cl}_{n}$ be the subalgebra of $\mathbb{H}_{W\left(\mathrm{~B}_{n}\right)}^{\mathrm{Cl}}$ generated by the elements $c_{i}$, which is isomorphic to the Clifford algebra. Let $U(n)$ be an irreducible supermodule of $\mathrm{Cl}_{n}$. The actions of $\mathbb{H}_{W\left(\mathrm{~B}_{n}\right)}^{\mathrm{Cl}}$ on $U(n) \widetilde{\otimes} U(n)$ are determined by the following:

$$
\begin{aligned}
& x_{i} \cdot(u \otimes v)=-(-1)^{\operatorname{deg}(u)} \sqrt{-1}\left(\left(\mathbf { k } _ { \alpha } \left(c_{1}+c_{2}+\cdots+c_{i-1}\right.\right.\right. \\
& \left.\left.\left.+(n-i) c_{i}\right)+\frac{\sqrt{2}}{2} \mathbf{k}_{\beta} c_{i}\right) \cdot u\right) \otimes\left(c_{i} \cdot v\right), \\
& s_{n} \cdot(u \otimes v)=(-1)^{\operatorname{deg}(u)} \sqrt{-1}\left(c_{n} \cdot u\right) \otimes\left(c_{n} \cdot v\right), \\
& s_{i, j} \cdot(u \otimes v)=(-1)^{\operatorname{deg}(u)} \sqrt{-1}\left(\frac{c_{i}-c_{j}}{\sqrt{2}} \cdot u\right) \otimes\left(\frac{c_{i}-c_{j}}{\sqrt{2}} \cdot v\right), \\
& c_{i} \cdot(u \otimes v)=(-1)^{\operatorname{deg}(u)}\left(u \otimes c_{i} \cdot v\right) .
\end{aligned}
$$

The above actions are indeed well defined: 
Proposition 5.1. For $N_{\mathrm{B}_{n}}=2(n-1) \mathbf{k}_{\alpha}^{2}+\sqrt{2} \mathbf{k}_{\alpha} \mathbf{k}_{\beta}$, the actions (5.16)-(5.18) above on $U(n) \widetilde{\otimes} U(n)$ define an $\mathbb{H}_{W\left(\mathrm{~B}_{n}\right)}^{\mathrm{Cl}}\left(\mathbf{k}, N_{\mathrm{B}_{n}}\right)$-module.

Proof. The computation is straightforward for verifying the defining relations of $\mathbb{H}_{W\left(\mathrm{~B}_{n}\right)}^{\mathrm{Cl}}$. For example,

$$
\begin{aligned}
&\left(s_{i, i+1} x_{i}-x_{i+1} s_{i, i+1}\right) \cdot(u \otimes v) \\
&=-\frac{1}{2} \mathbf{k}_{\alpha}\left(\left(-(n-i)+(n-i) c_{i} c_{i+1}\right) \cdot u\right) \otimes\left(\left(-1+c_{i} c_{i+1}\right) \cdot v\right) \\
&\left.+\frac{1}{2} \mathbf{k}_{\alpha}\left(\left((n-i-2)-(n-i) c_{i} c_{i+1}\right) \cdot u\right) \otimes\left(1-c_{i} c_{i+1}\right) \cdot v\right) \\
&= \mathbf{k}_{\alpha} u \otimes\left(\left(-1+c_{i} c_{i+1}\right) \cdot v\right) \\
&= \mathbf{k}_{\alpha}\left(-1+c_{i} c_{i+1}\right) \cdot(u \otimes v) .
\end{aligned}
$$

Moreover, for $i<j$, note that

$$
\begin{gathered}
\left(\mathbf{k}_{\alpha}\left(c_{1}+c_{2}+\cdots+c_{i-1}+(n-i) c_{i}\right)+\frac{\sqrt{2}}{2} \mathbf{k}_{\beta} c_{i}\right) \\
\cdot\left(\mathbf{k}_{\alpha}\left(c_{1}+c_{2}+\cdots+c_{j-1}+(n-j) c_{j}\right)+\frac{\sqrt{2}}{2} \mathbf{k}_{\beta} c_{j}\right) \\
+\left(\mathbf{k}_{\alpha}\left(c_{1}+c_{2}+\cdots+c_{j-1}+(n-j) c_{j}\right)+\frac{\sqrt{2}}{2} \mathbf{k}_{\beta} c_{j}\right) \\
\cdot\left(\mathbf{k}_{\alpha}\left(c_{1}+c_{2}+\cdots+c_{i-1}+(n-i) c_{i}\right)+\frac{\sqrt{2}}{2} \mathbf{k}_{\beta} c_{i}\right) \\
=-2(i-1) \mathbf{k}_{\alpha}^{2}+2 \mathbf{k}_{\alpha}\left(-(n-i) \mathbf{k}_{\alpha}-\frac{\sqrt{2}}{2} \mathbf{k}_{\beta}\right) \\
=-2(n-1) \mathbf{k}_{\alpha}^{2}-\sqrt{2} \mathbf{k}_{\alpha} \mathbf{k}_{\beta}
\end{gathered}
$$

and hence $x_{i} x_{j}-x_{j} x_{i}=\left(2(n-1) \mathbf{k}_{\alpha}^{2}+\sqrt{2} \mathbf{k}_{\alpha} \mathbf{k}_{\beta}\right) c_{j} c_{i}$. Other relations can be verified similarly (and more easily).

Denote the above $\mathbb{H}_{W\left(\mathrm{~B}_{n}\right)}^{\mathrm{Cl}}$-module by $\widetilde{\mathrm{St}_{\mathrm{B}_{n}}}$.

Type $\mathrm{D}_{n}$ : Set $N_{\mathrm{D}_{n}}=2(n-1) \mathbf{k}_{\alpha}^{2}$. Recall that $\mathbb{H}_{W\left(\mathrm{D}_{n}\right)}^{\mathrm{Cl}}$ is a subalgebra of the algebra $\mathbb{H}_{W\left(\mathrm{~B}_{n}\right)}^{\mathrm{Cl}}\left(\mathbf{k}^{B}, N_{\mathrm{D}_{n}}\right)$ (see $\mathbf{k}^{B}$ in Definition 4.18). By checking the parameter function, we have an $\mathbb{H}_{W\left(\mathrm{~B}_{n}\right)}^{\mathrm{Cl}}\left(\mathbf{k}^{B}, N_{\mathrm{D}_{n}}\right)$-module $\widetilde{\mathrm{St}}_{\mathrm{B}_{n}}$ defined above. Denote by $\widetilde{\mathrm{St}}_{\mathrm{D}_{n}}$ the restriction of $\widetilde{\mathrm{St}}_{\mathrm{B}_{n}}$ to an $\mathbb{H}_{W\left(\mathrm{D}_{n}\right)}^{\mathrm{Cl}}$-module.

\subsection{Dirac cohomology}

We keep using the notation in Section 5.

Proposition 5.2. Set $N_{\mathrm{B}_{n}}=2(n-1) \mathbf{k}_{\alpha}^{2}+\sqrt{2} \mathbf{k}_{\alpha} \mathbf{k}_{\beta}$ (with the notations in Section 5.1) and set $N_{\mathrm{D}_{n}}=2(n-1) \mathbf{k}^{2}$. Let $\mathbb{H}=\mathbb{H}_{\mathrm{A}_{n-1}}^{\mathrm{Cl}}, \mathbb{H}_{\mathrm{B}_{n}}^{\mathrm{Cl}}\left(\mathbf{k}, N_{\mathrm{B}_{n}}\right)$ or $\mathbb{H}_{\mathrm{D}_{n}}^{\mathrm{Cl}}\left(\mathbf{k}, N_{\mathrm{D}_{n}}\right)$. Let $X={\widetilde{\mathrm{St}}}_{\mathrm{A}_{n-1}}, \widetilde{\mathrm{St}}_{\mathrm{B}_{n}}$ or $\widetilde{\mathrm{St}}_{\mathrm{D}_{n}}$ be an $\mathbb{H}$-module defined in Section 5.1. The Dirac operator $D$ acts identically as zero on $X$. In particular, $H_{D}(X) \neq 0$. 
Proof. Type $\mathrm{A}_{n-1}$ : For $v \in \widetilde{\mathrm{St}}_{\mathrm{A}_{n-1}}$,

$$
\begin{aligned}
\pi(D) v & =\sum_{1 \leq j<i \leq n} s_{i j}\left(1-c_{i} c_{j}\right) c_{i} \cdot v+\sqrt{2} \mathbf{k} \sum_{\alpha \in R^{+}} \widetilde{s}_{\alpha} \cdot v \\
& =\sum_{1 \leq j<i \leq n} \mathbf{k} s_{i j}\left(c_{i}-c_{j}\right) \cdot v+\sqrt{2} \mathbf{k} \sum_{\alpha \in R^{+}} \widetilde{s}_{\alpha} \cdot v \\
& =\left(-\sqrt{2} \sum_{1<j<i<n} \mathbf{k} \widetilde{s}_{j i}+\sqrt{2} \mathbf{k} \sum_{\alpha \in R^{+}} \widetilde{s}_{\alpha}\right) \cdot v=0 .
\end{aligned}
$$

Type $\mathrm{B}_{n}$ : Recall that $\widetilde{\mathrm{St}}_{\mathrm{B}_{n}}$ is isomorphic to $U \widetilde{\otimes} U$ as vector spaces in the notation of Section 5.1. For $u \otimes v \in U \widetilde{\otimes} U$,

$$
\begin{aligned}
&(-1)^{\operatorname{deg}(u) \sqrt{-1}} \pi(D)(u \otimes v) \\
&= \sum_{i=1}^{n}\left(\left(\mathbf{k}_{\alpha}\left(c_{1}+c_{2}+\cdots+c_{i-1}+(n-i) c_{i}\right)+\frac{\sqrt{2}}{2} \mathbf{k}_{\beta} c_{i}\right) \cdot u\right) \otimes v \\
& \quad-\sqrt{2} \mathbf{k}_{\alpha} \sum_{1 \leq j<i \leq n}\left(\frac{c_{i}-c_{j}}{\sqrt{2}} \cdot u\right) \otimes v-\sqrt{2} \mathbf{k}_{\alpha} \sum_{1 \leq j<i \leq n}\left(\frac{c_{i}+c_{j}}{\sqrt{2}} \cdot u\right) \otimes v \\
& \quad-\frac{\sqrt{2}}{2} \mathbf{k}_{\beta} \sum_{i=1}^{n}\left(c_{i} . u\right) \otimes v \\
&= \sum_{i=1}^{n}\left(\left(2(n-i) c_{i}+\frac{\sqrt{2}}{2} \mathbf{k}_{\beta} c_{i}\right) \cdot u\right) \otimes v-\sqrt{2} \mathbf{k}_{\alpha} \sum_{i=1}^{n}\left(2(n-i) c_{i} . u\right) \otimes v \\
& \quad-\frac{\sqrt{2}}{2} \mathbf{k}_{\beta} \sum_{i=1}^{n}\left(c_{i} . u\right) \otimes v=0 .
\end{aligned}
$$

Type $\mathrm{D}_{n}$ : Recall that $\mathbb{H}_{W\left(\mathrm{D}_{n}\right)}^{\mathrm{Cl}}$ is a subalgebra of $\mathbb{H}_{W\left(\mathrm{~B}_{n}\right)}^{\mathrm{Cl}}\left(\mathbf{k}^{B}, N_{\mathrm{D}_{n}}\right)$ (see the notation of $\mathbf{k}^{B}$ in Definition 4.18). The Dirac operator for $\mathbb{H}_{W\left(\mathrm{D}_{n}\right)}^{\mathrm{Cl}}$ is the same as the Dirac operator for $\mathbb{H}_{W\left(\mathrm{~B}_{n}\right)}^{\mathrm{Cl}}\left(\mathbf{k}^{B}, N_{\mathrm{D}_{n}}\right)$. Then the vanishing result follows from the result for type $\mathrm{B}_{n}$, which has just been proven.

\section{Sergeev algebra}

The main purpose of this section is to review several results about Sergeev algebra, which will be useful for computing the Dirac cohomology of some modules for $\mathbb{H}_{W\left(\mathrm{~A}_{n-1}\right)}^{\mathrm{Cl}}$ in the next section. Some results can also be formulated to other types and one may refer to [WK, Sect. 2]. Starting from this section, we consider type $\mathrm{A}_{n-1}$ only and we shall usually use the notation $S_{n}$ for $W\left(\mathrm{~A}_{n-1}\right)$ (where $S_{n}$ represents the symmetric group). Write $R$ for $R\left(\mathrm{~A}_{n-1}\right)$ and $R^{+}$for $R^{+}\left(\mathrm{A}_{n-1}\right)$. Recall that $\Delta$ is the set of simple roots in $R$. 


\subsection{The superalgebra $\mathbb{C}\left[\widetilde{\boldsymbol{S}}_{n}\right]^{-}$}

Let $\widetilde{S}_{n}$ be the group generated by the elements $\psi, \widetilde{t}_{1,2}, \ldots, \widetilde{t}_{n-1, n}$ subject to the following relations:

$$
\begin{aligned}
\left(\widetilde{t}_{i, i+1}\right)^{2} & =1, \\
\left(\widetilde{t}_{i, i+1} \widetilde{t}_{i+1, i+2}\right)^{3} & =1 \quad \text { for } i=1, \ldots, n-1, \\
\widetilde{t}_{i, i+1} \widetilde{t}_{j, j+1} & =\psi \widetilde{t}_{j, j+1} \widetilde{t}_{i, i+1} \quad \text { for }|i-j|>1, \\
\psi \widetilde{t}_{i, i+1} & =\widetilde{t}_{i, i+1} \psi \quad \text { for } i=1, \ldots, n-1, \\
\psi^{2} & =1 .
\end{aligned}
$$

Then $\widetilde{S}_{n}$ is a double cover of $S_{n}$ via the map determined by sending $\widetilde{t}_{\alpha_{i}}$ to the transposition between $i$ and $i+1$, and $\psi \mapsto 1$. We also sometimes write $\widetilde{t}_{\alpha_{i, i+1}}$ for $t_{i, i+1}$ if we want to refer to the simple root $\alpha_{i, i+1}$. Denote by $\mathbb{C}\left[\widetilde{S}_{n}\right]$ the group algebra of $\widetilde{S}_{n}$ with a basis labeled as $\left\{e_{\widetilde{w}}: \widetilde{w} \in \widetilde{S}_{n}\right\}$. Define $\mathbb{C}\left[\widetilde{S}_{n}\right]^{-}:=$ $\mathbb{C}\left[\widetilde{S}_{n}\right] /\left\langle e_{\psi}+1\right\rangle$. We shall simply write $\widetilde{w}$ for the image of $e_{\widetilde{w}}$ in $\mathbb{C}\left[\widetilde{S}_{n}\right]^{-}$. There is a superalgebra structure on $\mathbb{C}\left[\widetilde{S}_{n}\right]^{-}$with $\operatorname{deg}\left(\widetilde{t}_{\alpha}\right)=1$ for all $\alpha \in \Delta$.

Lemma 6.1. Given an $S_{n}$-representation $U$ and a $\mathbb{C}\left[\widetilde{S}_{n}\right]^{-}$-module $U^{\prime}$, there exists a natural $\mathbb{C}\left[\widetilde{S}_{n}\right]^{-}$-module structure on $U \otimes U^{\prime}$ characterized by

$$
\widetilde{t}_{\alpha} \cdot\left(u \otimes u^{\prime}\right)=\left(s_{\alpha} \cdot u\right) \otimes\left(\widetilde{t}_{\alpha} \cdot u^{\prime}\right),
$$

where $\alpha \in \Delta, u \in U$, and $u^{\prime} \in U^{\prime}$.

Define an equivalence relation on $\operatorname{Irr}\left(\mathbb{C}\left[\widetilde{S}_{n}\right]^{-}\right): U \sim_{\text {sgn }} U^{\prime}$ if and only if $U=U^{\prime}$ or $U=\operatorname{sgn} \otimes U^{\prime}$ as $\mathbb{C}\left[\widetilde{S}_{n}\right]^{-}$-modules, where sgn is the sign representation of $S_{n}$ and the $\mathbb{C}\left[\widetilde{S}_{n}\right]^{-}$-module structure of $\operatorname{sgn} \otimes U^{\prime}$ is defined in Lemma 6.1.

Proposition 6.2. There is a natural bijection

$$
\operatorname{Irr}_{\text {sup }}\left(\mathbb{C}\left[\widetilde{S}_{n}\right]^{-}\right) / \sim_{\Pi} \longleftrightarrow \operatorname{Irr}\left(\mathbb{C}\left[\widetilde{S}_{n}\right]^{-}\right) / \sim_{\text {sgn }} .
$$

Proof. It suffices to see that the equivalence relation $\sim$ in Proposition 2.4 is the same as $\sim_{\text {sgn }}$. This follows from $\operatorname{deg}\left(\widetilde{t}_{\alpha}\right)=1$ for all $\alpha \in \Delta$ and definitions.

\subsection{Sergeev algebra}

Definition 6.3. Recall that $\mathbb{H}_{W\left(\mathrm{~A}_{n-1}\right)}^{\mathrm{Cl}}$ is defined in Definition 4.2. The Sergeev algebra, denoted $\mathrm{Seg}_{n}$, is the subalgebra of $\mathbb{H}_{W\left(\mathrm{~A}_{n-1}\right)}^{\mathrm{Cl}}$ generated by the elements $w \in W\left(\mathrm{~A}_{n-1}\right)=S_{n}$ and $c_{i}(i=1, \ldots, n)$. In other words, since $\mathbb{H}_{W\left(\mathrm{~A}_{n-1}\right)}^{\mathrm{Cl}}$ satisfies the property $(*), \operatorname{Seg}_{n}$ is the same as $\operatorname{Seg}\left(W_{\mathrm{A}_{n-1}}\right)$ in Definition 3.1. We shall use notations in Section 4.1 (e.g., $s_{\alpha}, c_{\alpha}, \widetilde{s}_{\alpha}$ ).

Let $\mathrm{Cl}_{n}$ be the supersubalgebra of $\operatorname{Seg}_{n}$ generated by $c_{i}(i=1, \ldots, n)$. There exists a unique, up to applying the functor $\Pi$, irreducible supermodule of $\mathrm{Cl}_{n}$. Let $U(n)$ be a fixed choice of an irreducible supermodule of $\mathrm{Cl}_{n}$. The dimension of $U(n)$ is $2^{n / 2}$ for $n$ even and $2^{(n+1) / 2}$ for $n$ odd.

The relation between subalgebras $\operatorname{Seg}_{n}$ and $\mathbb{C}\left[\widetilde{S}_{n}\right]^{-}$is the following. 
Lemma 6.4 ([Kl, Lem. 13.2.3]). $\mathrm{Seg}_{n}$ is isomorphic to $\mathbb{C}\left[\widetilde{S}_{n}\right]^{-} \widetilde{\otimes} \mathrm{Cl}_{n}$ as superalgebras.

Proof. Define a map:

$$
s_{\alpha} \mapsto \widetilde{t}_{\alpha} \otimes c_{\alpha} \quad(\alpha \in \Delta), \quad c_{i} \mapsto 1 \otimes c_{i} \quad(i=1, \ldots, n) .
$$

One can verify that the map is an isomorphism.

For any $\alpha \in R^{+}$, define $\widetilde{t}_{\alpha} \in \mathbb{C}\left[S_{n}\right]^{-}$such that $s_{\alpha}$ maps to $\widetilde{t}_{\alpha} \otimes c_{\alpha}$ under the map in the proof of Lemma 6.4.

Here is an analogue of Lemma 6.1:

Lemma 6.5. Given an $S_{n}$-representation $U$ and a $\mathrm{Seg}_{n}$-module $U^{\prime}$, there exists a natural $\mathrm{Seg}_{n}$-module structure on $U \otimes U^{\prime}$ characterized by

$$
s_{\alpha} \cdot\left(u \otimes u^{\prime}\right)=\left(s_{\alpha} \cdot u\right) \otimes\left(s_{\alpha} \cdot u^{\prime}\right)
$$

and

$$
c_{i} \cdot\left(u \otimes u^{\prime}\right)=u \otimes\left(c_{i} \cdot u^{\prime}\right),
$$

where $\alpha \in \Delta, i=1, \ldots, n, u \in U$, and $u^{\prime} \in U^{\prime}$.

\subsection{Relation between supermodules of $\mathbb{C}\left[\widetilde{\boldsymbol{S}}_{n}\right]^{-}$and $\operatorname{Seg}_{n}$}

Recall from $[\mathrm{BK}]$ (our formulation here is a bit different) a natural functor $F$ :

$$
\begin{aligned}
F: \operatorname{Mod}_{\text {sup }}\left(\mathbb{C}\left[\widetilde{S}_{n}\right]^{-}\right) & \rightarrow \operatorname{Mod}_{\text {sup }}\left(\operatorname{Seg}_{n}\right), \\
X & \mapsto X \otimes U(n) .
\end{aligned}
$$

The $\operatorname{Seg}_{n}$-supermodule structure of $X \otimes U(n)$ is characterized by

$$
\begin{gathered}
s_{\alpha} \cdot(x \otimes u)=-(-1)^{\operatorname{deg}(x)}\left(\widetilde{t}_{\alpha} \cdot x\right) \otimes\left(c_{\alpha} \cdot u\right) \quad(\alpha \in \Delta), \\
c_{i} \cdot(x \otimes u)=(-1)^{\operatorname{deg}(x)} x \otimes\left(c_{i} \cdot u\right) \quad(i=1, \ldots, n) .
\end{gathered}
$$

It is straightforward to check that the above equations define a $\operatorname{Seg}_{n}$-module. Next, define

$$
\begin{aligned}
G: \operatorname{Mod}_{\text {sup }}\left(\operatorname{Seg}_{n}\right) & \rightarrow \operatorname{Mod}_{\text {sup }}\left(\mathbb{C}\left[\widetilde{S}_{n}\right]^{-}\right), \\
Y & \mapsto \operatorname{Hom}_{\mathrm{Cl}_{n}}(U(n), Y)
\end{aligned}
$$

The $\mathbb{C}\left[\widetilde{S}_{n}\right]^{-}$-module structure is given by for $\theta \in \operatorname{Hom}_{\mathrm{Cl}_{n}}(U(n), Y)$,

$$
\left(\widetilde{t}_{\alpha} \cdot \theta\right)(u)=\left(s_{\alpha} c_{\alpha}\right) \cdot \theta(u) \quad(\alpha \in \Delta)
$$

Proposition 6.6 ([BK, Thm. 3.4]). The functors $F$ and $G$ form an adjoint pair, i.e., there is a natural isomorphism

$$
\operatorname{Hom}_{\mathrm{Seg}_{n}}\left(F(U), U^{\prime}\right)=\operatorname{Hom}_{\mathbb{C}\left[\widetilde{S}_{n}\right]^{-}}\left(U, G\left(U^{\prime}\right)\right) .
$$


Furthermore, if $n$ is even, $G \circ F=\operatorname{Id}$ and $F \circ G=\mathrm{Id}$. If $n$ is odd, $G \circ F=\operatorname{Id} \oplus \Pi$ and $F \circ G=\operatorname{Id} \oplus \Pi$, where $\Pi$ is defined in Section 2.2.

Let $U_{\mathrm{Cl}_{n}}$ be a $\mathrm{Seg}_{n}$-module defined by

$$
U_{\mathrm{Cl}_{n}}=\operatorname{Ind}_{\mathbb{C}\left[S_{n}\right]}^{\mathrm{Seg}_{n}} \text { triv }=\operatorname{Seg}_{n} \otimes_{\mathbb{C}\left[S_{n}\right]} \text { triv, }
$$

where $\mathbb{C}\left[S_{n}\right]$ is regarded as the subalgebra of $\operatorname{Seg}_{n}$ generated by the elements $f_{s_{\alpha}}$ for all $\alpha \in \Delta$ and triv is the trivial representation of $\mathbb{C}\left[S_{n}\right]$. In particular, $\operatorname{dim}_{\mathbb{C}} U_{\mathrm{Cl}_{n}}=2^{n}$.

We define a corresponding $\mathbb{C}\left[\widetilde{S}_{n}\right]^{-}$-module $U_{\text {spin }}$ as follows. If $n$ is even, define $U_{\text {spin }}=G\left(U_{\mathrm{Cl}_{n}}\right)$. If $n$ is odd, by [Kl, Prop. 13.2.2] and [Kl, Thm. 22.2.1], $G\left(U_{\mathrm{Cl}_{n}}\right)=$ $M \oplus \Pi(M)$ for some irreducible $\mathbb{C}\left[\widetilde{S}_{n}\right]^{-}$-module $M$. Then define $U_{\text {spin }}=M$.

An immediate consequence of Proposition 6.6 is given below.

Lemma 6.7. $F\left(U_{\text {spin }}\right)=U_{\mathrm{Cl}_{n}}$.

\section{Spectrum of the Dirac operator for type $A_{n-1}$}

We have seen the action of the Dirac operator on certain modules. In this section, we will go further for type $A_{n-1}$ and compute the action of $D$ on some interesting $\mathbb{H}_{W\left(\mathrm{~A}_{n-1}\right)}^{\mathrm{Cl}}$-modules. We shall see that Theorem 4.25 for $\mathbb{H}_{W\left(\mathrm{~A}_{n-1}\right)}^{\mathrm{Cl}}$ has interesting consequences. We shall write $\mathbb{H}_{n}^{\mathrm{Cl}}$ for $\mathbb{H}_{W\left(\mathrm{~A}_{n-1}\right)}^{\mathrm{Cl}}$ for simplicity. We keep using the notations in Section 4.1 and Section 6.

\subsection{Further notation for the root system of type $\mathbf{A}_{n-1}$}

A partition of $n$ is a sequence of positive integers $\left(n_{1}, \ldots, n_{r}\right)$ such that $n_{1} \geq$ $n_{2} \geq \ldots \geq n_{r}$ and $n_{1}+\cdots+n_{r}=n$. For a partition $\lambda=\left(n_{1}, \ldots, n_{r}\right)$ of $n$, let $I_{\lambda}=\{1, \ldots, n\} \backslash\left\{n_{1}, n_{1}+n_{2}, \ldots, n_{1}+\cdots+n_{r}\right\}$ and let

$$
\Delta_{\lambda}=\left\{e_{i}-e_{i+1}: i \in I_{\lambda}\right\} .
$$

Let $V_{\lambda}$ be the real span of $\Delta_{\lambda}$ in $\mathbb{R}^{n}$ and let $R_{\lambda}^{+}=V_{\lambda} \cap R^{+}$.

\subsection{Central characters for $\mathbb{H}_{n}^{\mathrm{Cl}}$}

The center of $\mathbb{H}_{n}^{\mathrm{Cl}}$ plays a role in the following computations.

Proposition 7.1 ([Kl, Thm. 14.3.1]). The center $Z\left(\mathbb{H}_{n}^{\mathrm{Cl}}\right)$ of $\mathbb{H}_{n}^{\mathrm{Cl}}$ is the set of all symmetric polynomials in $\mathbb{C}\left[x_{1}^{2}, x_{2}^{2}, \ldots, x_{n}^{2}\right]$. In particular, any element in $Z\left(\mathbb{H}_{n}^{\mathrm{Cl}}\right)$ is of even degree.

Definition 7.2. Recall that the central character $\chi_{\pi}: Z\left(\mathbb{H}_{n}^{\mathrm{Cl}}\right)_{0} \rightarrow \mathbb{C}$ of an irreducible supermodule $(\pi, X)$ is defined in Definition 2.6. By Proposition 7.1, we can also write $\chi_{\pi}: Z\left(\mathbb{H}_{n}^{\mathrm{Cl}}\right) \rightarrow \mathbb{C}$.

For an element $\gamma=\left(a_{1}, \ldots, a_{n}\right) \in \mathbb{C}^{n}$, define $\chi_{\gamma}^{\prime}: \mathbb{C}\left[x_{1}^{2}, \ldots, x_{n}^{2}\right] \rightarrow \mathbb{C}$ such that $\chi_{\gamma}^{\prime}\left(x_{i}^{2}\right)=a_{i}$. Define $\chi_{\gamma}$ to be the restriction of $\chi_{\gamma}^{\prime}$ to $Z\left(\mathbb{H}_{n}^{\mathrm{Cl}}\right)$. For the central character $\chi_{\pi}$ of $X$, there exists a unique $\gamma \in \mathbb{C}^{n}$, up to permutations of coordinates, such that $\chi_{\pi}=\chi_{\gamma}$. We may also say that $\gamma$ is the central character of $X$.

An $\mathbb{H}_{n}^{\mathrm{Cl}}$-module $(\pi, X)$ is said to be quasisimple if any element in $Z\left(\mathbb{H}_{n}^{\mathrm{Cl}}\right)$ acts by a scalar. In this case, $\gamma$ defined as above is still called the central character of $X$. 


\subsection{Induced modules}

Let us recall a construction of some $\mathbb{H}_{n}^{\mathrm{Cl}}$-modules in [HKS, Sect. 4], which is indeed modified from the module of type $\mathrm{A}_{n-1}$ in Section 5.1. There are also some similar constructions of $\mathbb{H}_{n}^{\mathrm{Cl}}$-modules in [Wa, Sect. 4]. Fix a partition $\lambda=\left(n_{1}, n_{2}, \ldots, n_{r}\right)$ of $n$. Let $S_{\lambda}$ be the subgroup of $S_{n}$ generated by $s_{i, i+1}$ for $i=\{1, \ldots, n\} \backslash$ $\left\{n_{1}, n_{1}+n_{2}, \ldots, n_{1}+\cdots+n_{r}\right\}$. It is easy to see that $S_{\lambda}$ is isomorphic to $S_{n_{1}} \times$ $\ldots \times S_{n_{r}}$. Let $\mathbb{H}_{\lambda}^{\mathrm{Cl}}$ be the supersubalgebra of $\mathbb{H}_{n}^{\mathrm{Cl}}$ generated by all $w \in S_{\lambda}, x_{i}$ $(i=1, \ldots, n)$ and $c_{i}(i=1, \ldots, n)$. Let $\operatorname{Seg}_{\lambda}$ be the supersubalgebra of $\mathbb{H}_{\lambda}^{C l}$ generated by all $w \in S_{\lambda}$ and $c_{i}(i=1, \ldots, n)$. Let $\widetilde{\mathrm{St}_{\lambda}}$ be an $\mathbb{H}_{\lambda}^{\mathrm{Cl}}$ module which is identified with $\mathrm{Cl}_{n}$ as vector spaces and the action of $\mathbb{H}_{\lambda}^{\mathrm{Cl}}$ is characterized by:

$$
\begin{gathered}
c_{i} .1=c_{i} \quad(i=1, \ldots, n), \quad s_{\alpha} \cdot 1=1 \quad\left(s_{\alpha} \in S_{\lambda}\right), \\
x_{i} \cdot v=\left(\sum_{n_{k-1}+1 \leq j<i \leq n_{k}} s_{i, j}\left(1-c_{i} c_{j}\right)\right) \cdot v \quad\left(i=n_{k}+1, \ldots, n_{k+1}\right),
\end{gathered}
$$

where $v$ is any vector in $\mathrm{Cl}_{n}$ and the actions of $s_{i, j}$ and $c_{i}, c_{j}$ are the ones defined in (5.14) and (5.15). It is straightforward to check that the above actions define an $\mathbb{H}_{\lambda}^{C l}$-module by verifying the defining relations of $\mathbb{H}_{\lambda}^{C l}$. Some details can be found in [HKS, Prop. 4.1.1].

Lemma 7.3. The element $x_{i}^{2}$ acts on $\widetilde{\mathrm{St}}_{\lambda}$ by a scalar $\left(i-n_{k}-1\right)\left(i-n_{k}\right)$ where $k=0, \ldots, r-1$ and $i=n_{k}+1, \ldots, n_{k+1}$.

Proof. Direct computation, or see [HKS, Prop. 4.1.1].

Define the Dirac-type element $\mathrm{D}_{\lambda}$ in $\mathbb{H}_{\lambda}^{\mathrm{Cl}}$ as:

$$
\mathrm{D}_{\lambda}=\sum_{i=1}^{n} y_{i}+\sqrt{2} \mathbf{k} \sum_{\alpha \in R_{\lambda}^{+}} \widetilde{s}_{\alpha}
$$

Proposition 7.4. The element $\mathrm{D}_{\lambda}$ acts as zero on the $\mathbb{H}_{\lambda}^{\mathrm{Cl}}$-module $\widetilde{\mathrm{St}}_{\lambda}$.

Proof. It follows a similar computation of type $\mathrm{A}_{n-1}$ in the proof of Proposition 5.2 .

Define

$$
X_{\lambda}=\operatorname{Ind}_{\mathbb{H}_{\lambda}^{\mathrm{Cl}}}^{\mathbb{H}_{n}^{\mathrm{Cl}}} \widetilde{\mathrm{St}}_{\lambda}=\mathbb{H}_{n}^{\mathrm{Cl}} \otimes_{\mathbb{H}_{\lambda}^{\mathrm{Cl}}} \widetilde{\mathrm{St}}_{\lambda}
$$

with the map $\pi_{\lambda}$ defining the action of $\mathbb{H}_{n}^{\mathrm{Cl}}$ on $X_{\lambda}$. Since $Z\left(\mathbb{H}_{n}^{\mathrm{Cl}}\right) \subset Z\left(\mathbb{H}_{\lambda}^{\mathrm{Cl}}\right)$, any element of $Z\left(\mathbb{H}_{n}^{\mathrm{Cl}}\right)$ acts by a scalar on $\widetilde{\mathrm{St}}_{\lambda}$. With the definitions for $X_{\lambda}$ and $Z\left(\mathbb{H}_{n}^{\mathrm{Cl}}\right)$, we have that $X_{\lambda}$ is quasisimple (Definition 7.2). The central character of $X_{\lambda}$ can be represented by

$$
(\underbrace{1(1-1), \ldots, n_{1}\left(n_{1}-1\right)}_{n_{1} \text { terms }}, \ldots, \underbrace{1(1-1), \ldots, n_{r}\left(n_{r}-1\right)}_{n_{r} \text { terms }}) \in \mathbb{R}^{n} .
$$

To compute the Dirac cohomology of the above induced modules, we need some more information discussed in the next subsections. 


\section{4. $S_{n}$-structure and $\operatorname{Seg}_{n}$-structure of $\left(\pi_{\lambda}, X_{\lambda}\right)$}

We continue to fix a partition $\lambda$ of $n$. Recall that in Definition 4.2(1), $\mathbb{H}_{n}^{\mathrm{Cl}}$ contains $\mathbb{C}\left[S_{n}\right]$ as a subalgebra. Let $\left(\pi_{V}, V=\mathbb{C}^{n}\right)$ be the $S_{n}$-representation such that elements in $S_{n}$ permute the coordinates.

Lemma 7.5. The restriction of $X_{\lambda}$ to $\mathbb{C}\left[S_{n}\right]$ is isomorphic to

$$
\mathbb{C}\left[S_{n}\right] \otimes_{\mathbb{C}\left[S_{\lambda}\right]} \operatorname{Res}_{\mathbb{C}\left[S_{\lambda}\right]}^{\mathbb{C}\left[S_{n}\right]}\left(\bigoplus_{i=0}^{n} \wedge^{i} V\right)
$$

as $\mathbb{C}\left[S_{n}\right]$-modules.

Proof. Note that the restriction of $\widetilde{\mathrm{St}_{\lambda}}$ to $\mathbb{C}\left[S_{\lambda}\right]$ is isomorphic to $\operatorname{Res}_{\mathbb{C}\left[S_{\lambda}\right]}^{\mathbb{C}\left[S_{n}\right]}\left(\bigoplus_{i=0}^{n} \wedge^{i} V\right)$. Then $\mathbb{H}_{n}^{\mathrm{Cl}} \otimes_{\mathbb{H}_{\lambda}^{\mathrm{Cl}}} \widetilde{\mathrm{St}}_{\lambda}$ and $\mathbb{C}\left[S_{n}\right] \otimes_{\mathbb{C}\left[S_{\lambda}\right]} \operatorname{Res}_{\mathbb{C}\left[S_{\lambda}\right]}^{\mathbb{C}\left[S_{n}\right]}\left(\bigoplus_{i=0}^{n} \wedge^{i} V\right)$ are isomorphic as $\mathbb{C}\left[S_{n}\right]$ modules.

It is well known that we have the following $\mathbb{C}\left[S_{n}\right]$-isomorphism:

$$
\mathbb{C}\left[S_{n}\right] \otimes_{\mathbb{C}\left[S_{\lambda}\right]} \operatorname{Res}_{S_{\lambda}}^{S_{n}}\left(\bigoplus_{i=0}^{n} \wedge^{i} V\right) \cong\left(\mathbb{C}\left[S_{n}\right] \otimes_{\mathbb{C}\left[S_{\lambda}\right]} \text { triv }\right) \otimes \bigoplus_{i=0}^{n} \wedge^{i} V .
$$

Here the module in the right-hand side is viewed as the tensor product of two $S_{n}$-representations. The isomorphism is given by

$$
w \otimes\left(v_{1} \wedge \cdots \wedge v_{i}\right) \mapsto(w \otimes 1) \otimes\left(\pi_{V}(w) v_{1} \wedge \cdots \wedge \pi_{V}(w) v_{i}\right) .
$$

Note that the space $\oplus_{i=0}^{n} \wedge^{i} V$ can be identified with $\mathrm{Cl}_{n}$ via the map determined by

$$
e_{i_{1}} \wedge \cdots \wedge e_{i_{r}} \mapsto c_{i_{1}} \cdots c_{i_{r}},
$$

where $\left\{e_{1}, \ldots, e_{n}\right\}$ is the standard basis of $V=\mathbb{C}^{n}$. Thus $X_{\lambda}=\operatorname{Ind}_{\mathbb{H}_{\lambda}^{\mathrm{Cl}}}^{\mathbb{H}^{\mathrm{Cl}}} \widetilde{\mathrm{St}}_{\lambda}$ can be identified with, as vector spaces, $\left(\mathbb{C}\left[S_{n}\right] \otimes_{\mathbb{C}\left[S_{\lambda}\right]}\right.$ triv $) \otimes U_{\mathrm{Cl}_{n}}$ via the identification in Lemma 7.5 and the above identification between $\oplus_{i=1}^{n} \wedge^{i} V$ and $\mathrm{Cl}_{n}$. Then if we translate the action of the subalgebra $\operatorname{Seg}_{n}$ under the above identifications, then we have:

$$
\begin{aligned}
& \pi_{\lambda}(w)\left(w^{\prime} \otimes 1 \otimes c_{i_{1}} \ldots c_{i_{r}}\right)=w w^{\prime} \otimes 1 \otimes c_{w\left(i_{1}\right)} \ldots c_{w\left(i_{r}\right)}, \\
& \pi_{\lambda}\left(c_{i}\right)\left(w^{\prime} \otimes 1 \otimes c_{i_{1}} \ldots c_{i_{r}}\right)=w^{\prime} \otimes 1 \otimes c_{i} c_{i_{1}} \ldots c_{i_{r}} .
\end{aligned}
$$

We have just proven that:

Lemma 7.6. As $\mathrm{Seg}_{n}$-supermodules,

$$
\operatorname{Res}_{\operatorname{Seg}_{n}}^{\mathbb{H}_{n}^{\mathrm{Cl}}} X_{\lambda}=\left(\mathbb{C}\left[S_{n}\right] \otimes_{\mathbb{C}\left[S_{\lambda}\right]} \text { triv }\right) \otimes U_{\mathrm{Cl}_{n}},
$$

where the supermodule in the right hand side has the $\mathrm{Seg}_{n}$-supermodule structure described in Lemma 6.5 .

Recall that $F$ is the functor defined in Section 6.3. 
Proposition 7.7. As $\mathrm{Seg}_{n}$-supermodules,

$$
\operatorname{Res}_{\mathrm{Seg}_{n}}^{\mathbb{H}_{n}^{\mathrm{Cl}}} X_{\lambda}=F\left(\left(\mathbb{C}\left[S_{n}\right] \otimes_{\mathbb{C}\left[S_{\lambda}\right]} \text { triv }\right) \otimes U_{\text {spin }}\right)
$$

where $\left(\mathbb{C}\left[S_{n}\right] \otimes_{\mathbb{C}\left[S_{\lambda}\right]}\right.$ triv $) \otimes U_{\text {spin }}$ has $\mathbb{C}\left[\widetilde{S}_{n}\right]^{-}$-supermodule described in Lemma 6.1 . Proof. By Lemma 7.6, it suffices to show

$$
\left(\mathbb{C}\left[S_{n}\right] \otimes_{\mathbb{C}\left[S_{\lambda}\right]} \text { triv }\right) \otimes U_{\mathrm{Cl}_{n}}=\left(\mathbb{C}\left[S_{n}\right] \otimes_{\mathbb{C}\left[S_{\lambda}\right]} \text { triv }\right) \otimes U_{\text {spin }} \otimes U(n) .
$$

By Lemma 6.7, there is a $\operatorname{Seg}_{n}$-module isomorphism $f$ from $U_{\mathrm{Cl}_{n}}$ to $F\left(U_{\text {spin }}\right)=$ $U_{\text {spin }} \otimes U(n)$. Then define a vector space isomorphism of $\operatorname{Seg}_{n}$-modules

$$
\left(\mathbb{C}\left[S_{n}\right] \otimes_{\mathbb{C}\left[S_{\lambda}\right]} \text { triv }\right) \otimes U_{\mathrm{Cl}_{n}} \rightarrow\left(\mathbb{C}\left[S_{n}\right] \otimes_{\mathbb{C}\left[S_{\lambda}\right]} \text { triv }\right) \otimes U_{\text {spin }} \otimes U(n)
$$

determined by

$$
(w \otimes 1) \otimes\left(c_{i_{1}} \ldots c_{i_{r}} \otimes 1\right) \mapsto(w \otimes 1) \otimes f\left(c_{i_{1}} \ldots c_{i_{r}} \otimes 1\right) .
$$

Using the module structure described before Lemma 7.6, one can check the linear isomorphism is $\mathrm{Seg}_{n}$-equivariant.

\subsection{Hermitian form on $\left(\pi_{\lambda}, X_{\lambda}\right)$}

We continue to fix a partition $\lambda$ of $n$. In this subsection, we shall construct a Hermitian form on the $\mathbb{H}_{n}^{\mathrm{Cl}}$-module $\left(\pi_{\lambda}, X_{\lambda}\right)$ such that the adjoint operator of $\pi_{\lambda}(D)$ with respect to such form is $-\pi_{\lambda}(D)$. We will see this makes the computation for the Dirac cohomology $H_{D}(X)$ of those modules $X$ much easier.

Recall that $\mathrm{Seg}_{\lambda}$ is a subalgebra of $\mathbb{H}_{\lambda}^{\mathrm{Cl}}$.

Lemma 7.8. There exists a $\mathrm{Seg}_{\lambda}$-invariant positive definite Hermitian form on $\widetilde{\mathrm{St}_{\lambda}}$.

Proof. Since $\operatorname{Res}_{\mathrm{Seg}_{\lambda}}^{\mathbb{H}_{\lambda}^{\mathrm{Cl}}} \widetilde{\mathrm{St}_{\lambda}}=\operatorname{Res}_{\mathrm{Seg}_{\lambda}}^{\operatorname{Seg}_{n}} U_{\mathrm{Cl}_{n}}$ as $\operatorname{Seg}_{\lambda}$-modules, it suffices to consider the case when $\lambda=(n)$. Recall that $U_{\mathrm{Cl}_{n}}=\operatorname{Seg}_{n} \otimes_{\mathbb{C}\left[S_{n}\right]}$ triv in Section 6.3. Define $\langle\cdot, \cdot\rangle: U_{\mathrm{Cl}_{n}} \times U_{\mathrm{Cl}_{n}} \rightarrow \mathbb{C}$ such that for $1 \leq i_{1}<\cdots<i_{r} \leq n$ and $1 \leq j_{1}<\cdots<$ $j_{s} \leq n$,

$$
\left\langle c_{i_{1}} c_{i_{2}} \cdots c_{i_{r}} \otimes 1, c_{j_{1}} c_{j_{2}} \cdots c_{j_{s}} \otimes 1\right\rangle= \begin{cases}1 & \text { if }\left\{i_{1}, \ldots, i_{r}\right\}=\left\{j_{1}, \ldots, j_{s}\right\} \\ 0 & \text { otherwise }\end{cases}
$$

It is straightforward to check that $\langle$,$\rangle satisfies the desired properties.$

We denote the $\operatorname{Seg}_{\lambda}$-invariant Hermitian form on $\widetilde{\mathrm{St}}_{\lambda}$ in the above lemma by $\langle\cdot, \cdot\rangle_{\lambda}$. Recall that $X_{\lambda}=\mathbb{H}_{n}^{\mathrm{Cl}} \otimes_{\mathbb{H}_{\lambda}^{\mathrm{Cl}}} \widetilde{\mathrm{St}_{\lambda}}$. We define a bilinear form $\langle\cdot, \cdot\rangle$ on $X_{\lambda}$ characterized by:

$$
\left\langle w_{1} \otimes v_{1}, w_{2} \otimes v_{2}\right\rangle=\delta_{w_{1} S_{\lambda}, w_{2} S_{\lambda}}\left\langle\pi_{\lambda}\left(w_{2}^{-1} w_{1}\right) v_{1}, v_{2}\right\rangle_{\lambda}
$$

where $w_{1}, w_{2} \in S_{n}$ and $\delta_{w_{1} S_{\lambda}, w_{2} S_{\lambda}}=1$ if $w_{1} S_{\lambda}=w_{2} S_{\lambda}$ and $\delta_{w_{1} S_{\lambda}, w_{2} S_{\lambda}}=0$ otherwise. 
Lemma 7.9. $\langle\cdot, \cdot\rangle$ defined above is a positive definite Hermitian form.

Proof. This follows from the property that $\langle\cdot, \cdot\rangle_{\lambda}$ is positive definite and Hermitian.

We next compute the adjoint operator of $\pi_{\lambda}(D)$ with respect to $\langle\cdot, \cdot\rangle$. We begin with some lemmas.

Lemma 7.10. For $v_{1}, v_{2} \in \widetilde{\mathrm{St}}_{\lambda},\left\langle D \otimes v_{1}, 1 \otimes v_{2}\right\rangle=\left\langle 1 \otimes v_{1}, D \otimes v_{2}\right\rangle=0$.

Proof. For $\alpha \in R^{+} \backslash R_{\lambda}^{+}$, one has

$$
\left\langle\widetilde{s}_{\alpha} \otimes v_{1}, 1 \otimes v_{2}\right\rangle=0
$$

With the equality

$$
D=D_{\lambda}+\sqrt{2} \mathbf{k} \sum_{\alpha>0, \alpha \in R^{+} \backslash R_{\lambda}^{+}} \widetilde{s}_{\alpha},
$$

one has $\left\langle D \otimes v_{1}, 1 \otimes v_{2}\right\rangle=\left\langle D_{\lambda} . v_{1}, v_{2}\right\rangle_{\lambda}$. Then we have $\left\langle D \otimes v_{1}, 1 \otimes v_{2}\right\rangle=0$ by Proposition 7.4. The proof for $\left\langle 1 \otimes v_{1}, D \otimes v_{2}\right\rangle=0$ is similar.

Lemma 7.11. Suppose $\beta_{1} \neq \beta_{2}$ and $\beta_{1}, \beta_{2} \in R^{+} \backslash R_{\lambda}^{+}$. Then $s_{\beta_{1}} s_{\beta_{2}} \notin S_{\lambda}$.

Proof. In the following, we implicitly use several times the fact that any element in $S_{\lambda}$ cannot send a positive root not in $R_{\lambda}$ to a negative root. If $\left\langle\beta_{1}, \beta_{2}\right\rangle=0$, then $s_{\beta_{1}} s_{\beta_{2}}\left(\beta_{2}\right)=-\beta_{2}<0$. Since $\beta_{2} \notin R_{\lambda}, s_{\beta_{1}} s_{\beta_{2}} \notin S_{\lambda}$. If $\left\langle\beta_{1}, \beta_{2}\right\rangle=-1$, then $s_{\beta_{2}}\left(\beta_{1}\right)=\beta_{1}+\beta_{2}>0$. Moreover, $s_{\beta_{1}} s_{\beta_{2}}\left(s_{\beta_{2}}\left(\beta_{1}\right)\right)=-\beta_{1}<0$. Since $\beta_{1}+\beta_{2} \notin R_{\lambda}$, $s_{\beta_{1}} s_{\beta_{2}} \notin S_{\lambda}$. If $\left\langle\beta_{1}, \beta_{2}\right\rangle=1$, then either $s_{\beta_{1}}\left(\beta_{2}\right)>0$ or $s_{\beta_{2}}\left(\beta_{1}\right)>0$. In the case that $s_{\beta_{1}}\left(\beta_{2}\right)>0, s_{\beta_{1}} s_{\beta_{2}}\left(\beta_{2}\right)=-s_{\beta_{1}}\left(\beta_{2}\right)<0$. Then since $\beta_{2} \notin R_{\lambda}, s_{\beta_{1}} s_{\beta_{2}} \notin S_{\lambda}$. A similar argument by considering $\left(s_{\beta_{1}} s_{\beta_{2}}\right)^{-1}$ can prove another case.

Proposition 7.12. The adjoint operator of $\pi_{\lambda}(D)$ with respect to $\langle\cdot, \cdot\rangle$ is $-\pi_{\lambda}(D)$.

Proof. It suffices to show that

$$
\left\langle D w_{1} \otimes v_{1}, w_{2} \otimes v_{2}\right\rangle=\left\langle w_{1} \otimes v_{1},-D w_{2} \otimes v_{2}\right\rangle
$$

for any $w_{1}, w_{2} \in S_{n}$ and $v_{1}, v_{2} \in X_{\lambda}$. To this end, we consider two cases. Suppose $w_{1} S_{\lambda}=w_{2} S_{\lambda}$. Then,

$$
\begin{aligned}
\left\langle D w_{1} \otimes v_{1}, w_{2} \otimes v_{2}\right\rangle & =\left\langle w_{2}^{-1} D w_{1} \otimes v_{1}, 1 \otimes v_{2}\right\rangle \\
& =\left\langle D w_{2}^{-1} w_{1} \otimes v_{1}, 1 \otimes v_{2}\right\rangle \\
& =\left\langle D \otimes\left(w_{2}^{-1} w_{1}\right) \cdot v_{1}, 1 \otimes v_{2}\right\rangle \\
& =0 \quad(\text { by Lemma } 7.10) .
\end{aligned}
$$

Similarly, we also have

$$
\left\langle w_{1} \otimes v_{1}, D w_{2} \otimes v_{2}\right\rangle=0 .
$$

and so $\left\langle D w_{1} \otimes v_{1}, w_{2} \otimes v_{2}\right\rangle=\left\langle w_{1} \otimes v_{1},-D w_{2} \otimes v_{2}\right\rangle$. 
Now we suppose that $w_{1} S_{\lambda} \neq w_{2} S_{\lambda}$. Without loss of generality, assume that $w_{2}^{-1} w_{1}$ is a minimal representative in $w_{2}^{-1} w_{1} S_{\lambda}$.

$$
\begin{aligned}
\left\langle w_{2}^{-1} w_{1} D \otimes v_{1}, 1 \otimes v_{2}\right\rangle= & \left\langle w_{2}^{-1} w_{1} \sqrt{2} \sum_{\alpha>0} \mathbf{k} \widetilde{s}_{\alpha} \otimes v_{1}, 1 \otimes v_{2}\right\rangle \\
= & \left\langle 1 \otimes v_{1}, \sqrt{2} \sum_{\alpha>0} \mathbf{k} \widetilde{s}_{\alpha} w_{1}^{-1} w_{2} \otimes v_{2}\right\rangle \\
= & -\left\langle 1 \otimes v_{1}, D w_{1}^{-1} w_{2} \otimes v_{2}\right\rangle+\left\langle 1 \otimes v_{1}, w_{1}^{-1} w_{2} D \otimes v_{2}\right\rangle \\
& +\left\langle 1 \otimes v_{1}, \sqrt{2} \sum_{\alpha>0} \mathbf{k} \widetilde{s}_{\alpha} w_{1}^{-1} w_{2} \otimes v_{2}\right\rangle
\end{aligned}
$$

It remains to show

$$
\left\langle 1 \otimes v_{1}, w_{1}^{-1} w_{2} D \otimes v_{2}\right\rangle+\sqrt{2}\left\langle 1 \otimes v_{1}, \sum_{\alpha>0} \mathbf{k} \widetilde{s}_{\alpha} w_{1}^{-1} w_{2} \otimes v_{2}\right\rangle=0
$$

By Lemma 7.11, there exists at most one $\beta \in R^{+} \backslash R_{\lambda}$ such that $w_{1}^{-1} w_{2} s_{\beta} \in S_{\lambda}$. If such $\beta$ does not exist, then the two terms in the left-hand side of the above equation are both zero and so the equation holds. If such unique $\beta$ exists, let $\beta^{\prime}=-w_{1}^{-1} w_{2}(\beta)$. Note that $\beta^{\prime}>0$, otherwise $w_{1}^{-1} w_{2} s_{\beta} \notin S_{\lambda}$. Then

$$
\begin{aligned}
\left\langle 1 \otimes v_{1}, w_{1}^{-1} w_{2} D \otimes\right. & \left.v_{2}\right\rangle+\sqrt{2}\left\langle 1 \otimes v_{1}, \sum_{\alpha>0} \mathbf{k} \widetilde{s}_{\alpha} w_{1}^{-1} w_{2} \otimes v_{2}\right\rangle \\
= & \sqrt{2} \mathbf{k}\left\langle 1 \otimes v_{1}, w_{1}^{-1} w_{2} \widetilde{s}_{\beta} \otimes v_{2}\right\rangle \\
& \left.+\sqrt{2} \mathbf{k}\left\langle 1 \otimes v_{1}, \widetilde{s}_{\beta^{\prime}} w_{1}^{-1} w_{2} \otimes v_{2}\right\rangle \text { (by definition of } D \text { and }\langle\cdot, \cdot\rangle\right) \\
= & -\sqrt{2} \mathbf{k}\left\langle 1 \otimes v_{1}, \widetilde{s}_{\beta^{\prime}} w_{1}^{-1} w_{2} \otimes v_{2}\right\rangle \\
& \left.+\sqrt{2} \mathbf{k}\left\langle 1 \otimes v_{1}, \widetilde{s}_{\beta^{\prime}} w_{1}^{-1} w_{2} \otimes v_{2}\right\rangle \quad \text { (by Lemma } 4.4(4)\right) \\
= & 0 .
\end{aligned}
$$

This completes the proof.

Proposition 7.13. Let $\left(\pi_{\lambda}, X_{\lambda}\right)$ be the $\mathbb{H}_{n}^{\mathrm{Cl}}$-module as in (7.19). Then

$$
\operatorname{ker} \pi_{\lambda}(D)=\operatorname{ker} \pi_{\lambda}\left(D^{2}\right)
$$

and

$$
\operatorname{ker} \pi_{\lambda}(D) \cap \operatorname{im} \pi_{\lambda}(D)=0 .
$$

In particular, $H_{D}\left(X_{\lambda}\right)=\operatorname{ker} \pi_{\lambda}\left(D^{2}\right)$.

Proof. It is clear that $\operatorname{ker} \pi_{\lambda}(D) \subset \operatorname{ker} \pi_{\lambda}\left(D^{2}\right)$. For $v \in \operatorname{ker} \pi_{\lambda}\left(D^{2}\right)$, we have $\left\langle\pi_{\lambda}(D) v,-\pi_{\lambda}(D) v\right\rangle=\left\langle\pi_{\lambda}\left(D^{2}\right) v, v\right\rangle=0$ by Proposition 7.12 . Since $\langle\cdot, \cdot\rangle$ is positive definite by Lemma $7.9, \pi_{\lambda}(D) v=0$. This proves the first equation, $\operatorname{ker} \pi_{\lambda}(D)=$ $\operatorname{ker} \pi_{\lambda}\left(D^{2}\right)$. The equation $\operatorname{ker} \pi_{\lambda}(D) \cap \operatorname{im} \pi_{\lambda}(D)=0$ follows from the first one. 


\subsection{Dirac cohomology of $\boldsymbol{X}_{\boldsymbol{\lambda}}$}

Let $\mathcal{P}_{n}$ be the set of partitions of $n$. One can attach an element in $\mathcal{P}_{n}$ to a point in $\mathbb{R}^{n}$ via the Jacobson-Morozov triple. The map, denoted $\Phi_{1}: \mathcal{P}_{n} \rightarrow \mathbb{R}^{n}$ can be explicitly described as:

$$
\left(n_{1}, n_{2}, \ldots, n_{r}\right) \mapsto(\underbrace{-n_{1}+1,-n_{1}+3, \ldots, n_{1}-1}_{n_{1} \text { terms }}, \ldots, \underbrace{-n_{r}+1,-n_{r}+3, \ldots, n_{r}-1}_{n_{r} \text { terms }}) .
$$

There is another way to attach an element in $\mathcal{P}_{n}$ to a point in $\mathbb{R}^{n}$ via the central characters of the modules $X_{\lambda}$. This map, denoted $\Phi_{2}: \mathcal{P}_{n} \rightarrow \mathbb{R}^{n}$, is:

$$
\left(n_{1}, n_{2}, \ldots, n_{r}\right) \mapsto(\underbrace{\sqrt{(1-1) 1}, \ldots, \sqrt{\left(n_{1}-1\right) n_{1}}}_{n_{1} \text { terms }}, \ldots, \underbrace{\left.\sqrt{(1-1) 1}, \ldots, \sqrt{n_{r}\left(n_{r}-1\right)}\right)}_{n_{r} \text { terms }} .
$$

The first interesting computational fact is the following:

Lemma 7.14. For a partition $\lambda$ of $n,\left|\Phi_{1}(\lambda)\right|=\left|\Phi_{2}(\lambda)\right|$, where $|\cdot|$ denotes the standard Euclidean norm in $\mathbb{R}^{n}$.

Proof. This follows from the computation that

$$
\sum_{k=1}^{n_{i}}\left(-n_{i}+2 k-1\right)^{2}=\sum_{k=1}^{n_{i}} k(k-1)=\frac{1}{3}\left(n_{i}-1\right) n_{i}\left(n_{i}+1\right) .
$$

For each $\lambda \in \mathcal{P}_{n}$, define a $S_{n}$-representation:

$$
W_{\lambda}=\left(\operatorname{Ind}_{\mathbb{C}\left[S_{\lambda}\right]}^{\mathbb{C}\left[S_{n}\right]} \operatorname{triv}\right) \cap\left(\operatorname{Ind}_{\mathbb{C}\left[S_{\lambda^{t}}\right]}^{\mathbb{C}\left[S_{n}\right]} \operatorname{sgn}\right)
$$

where sgn and triv are respectively the sign and trivial representations of $S_{\lambda}$, and $\lambda^{t}$ is the conjugate of $\lambda$. It is well known that $W_{\lambda}$ exhausts the list of irreducible representations of $S_{n}$.

Define

$$
\Omega_{\mathbb{C}\left[\widetilde{S}_{n}\right]^{-}}=\underset{\substack{\alpha>0, \beta>0, s_{\alpha}(\beta)<0 \\ 2}}{ } \widetilde{t}_{\alpha} \widetilde{t}_{\beta} \in \mathbb{C}\left[\widetilde{S}_{n}\right]^{-}
$$

Let $P_{n}^{\text {dist }}$ be the set of partitions of $n$ with distinct parts. Recall that we denote by $\operatorname{Irr}_{\text {sup }} \mathbb{C}\left[\widetilde{S}_{n}\right]^{-}$(resp. $\operatorname{Irr}_{\text {sup }} \operatorname{Seg}_{n}$ ) the set of irreducible supermodules of $\mathbb{C}\left[\widetilde{S}_{n}\right]^{-}$(resp. Seg $\left.{ }_{n}\right)$. Recall that the equivalence relation $\sim_{\Pi}$ on $\operatorname{Irr}_{\text {sup }} \mathbb{C}\left[\widetilde{S}_{n}\right]^{-}$or $\operatorname{Irr}_{\text {sup }} \operatorname{Seg}_{n}$ is defined in Section 2.3.

Proposition 7.15 ([Ci1, Part of Thm. 1.0.1] (also see [St])). There exists a bijection $\Psi_{1}: \mathcal{P}_{n}^{\text {dist }} \rightarrow \operatorname{Irr}_{\text {sup }} \mathbb{C}\left[\widetilde{S}_{n}\right]^{-} / \sim_{\Pi}$ such that for each partition $\lambda$ of $n$, there exists a representative $(\sigma, U) \in \Psi_{1}(\lambda)$ with the properties that

$$
\mathbf{k}^{2}\left|\Phi_{1}(\lambda)\right|^{2}=\chi_{\sigma}\left(\Omega_{\mathbb{C}_{\left[\widetilde{S}_{n}\right]^{-}}}\right)
$$

and

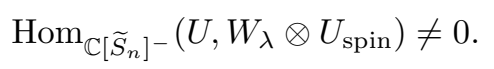


Proof. In [Ci1, Thm. 1.0.1], the set $\operatorname{Irr} \mathbb{C}\left[\widetilde{S}_{n}\right]^{-} / \sim_{\text {sgn }}$ is considered instead of the set $\operatorname{Irr}_{\text {sup }} \mathbb{C}\left[\widetilde{S}_{n}\right]^{-} / \sim_{\Pi}$. By Proposition 6.2 , there is a natural bijection between $\operatorname{Irr} \mathbb{C}\left[\widetilde{S}_{n}\right]^{-} / \sim_{\text {sgn }}$ and $\operatorname{Irr}_{\text {sup }} \mathbb{C}\left[\widetilde{S}_{n}\right]^{-} / \sim_{\Pi}$. Then one can now apply [Ci1, Thm. 1.0.1].

Here is an analogue of Proposition 7.15. Recall that $\Omega_{\operatorname{Seg}_{n}}$ (i.e., $\Omega_{\mathrm{Seg}\left(W_{\mathrm{A}_{n-1}}\right)}$ ) is defined in Theorem 4.23.

Proposition 7.16. There exists a bijection $\Psi_{2}: \mathcal{P}_{n}^{\text {dist }} \rightarrow \operatorname{Irr}_{\text {sup }} \operatorname{Seg}_{n} / \sim_{\Pi}$ such that there exists a representative $(\sigma, U) \in \Psi_{2}(\lambda)$ with the properties that

$$
\mathbf{k}^{2}\left|\Phi_{2}(\lambda)\right|^{2}=\chi_{\sigma}\left(\Omega_{\mathrm{Seg}_{n}}\right)
$$

and

$$
\operatorname{Hom}_{\operatorname{Seg}_{n}}\left(U, F\left(W_{\lambda} \otimes U_{\text {spin }}\right)\right) \neq 0 .
$$

Proof. Note that for an irreducible $\mathbb{C}\left[\widetilde{S}_{n}\right]^{-}$-supermodule $U, F(U)$ is either an irreducible supermodule or the direct sum of two irreducible supermodules of opposite grading. Thus we could define $\Psi_{2}(\lambda)$ to be the unique equivalence class in $\operatorname{Irr}_{\text {sup }} \operatorname{Seg}_{n} / \sim_{\Pi}$ containing the irreducible supermodule(s) in $F(U)$ for a representative $U \in \Phi_{1}(\lambda)$, where $\Phi_{1}$ is defined in Proposition 7.15.

It remains to check those two properties. Recall that $F(U)=U \otimes U(n)$ and that the action of $\operatorname{Seg}_{n}$ on $F(U)$ is defined in Section 6.3. Then for $u \otimes u^{\prime} \in U \otimes U(n)$,

$$
\begin{aligned}
\Omega_{\operatorname{Seg}_{n}} \cdot\left(u \otimes u^{\prime}\right) & =2 \mathbf{k}^{2} \sum_{\alpha, \beta>0, s_{\alpha}(\beta)<0} \widetilde{s}_{\alpha} \widetilde{s}_{\beta} \cdot\left(u \otimes u^{\prime}\right) \\
& =2 \mathbf{k}^{2}\left(\sum_{\alpha, \beta>0, s_{\alpha}(\beta)<0} \widetilde{t}_{\alpha} \widetilde{t}_{\beta} \cdot u\right) \otimes u^{\prime} \\
& =\chi_{\sigma}\left(\Omega_{\left.\mathbb{C}_{C} \widetilde{S}_{n}\right]^{-}}\right) u \otimes u^{\prime} .
\end{aligned}
$$

Thus for any irreducible supermodule $\left(\sigma^{\prime}, U^{\prime}\right)$ in $F(U), \chi_{\sigma^{\prime}}\left(\Omega_{\operatorname{Seg}_{n}}\right)=\chi_{\sigma}\left(\Omega_{\left.\mathbb{C}_{[} \widetilde{S}_{n}\right]^{-}}\right)$. Then combining this with Lemma 7.14 and Proposition 7.15, we have shown the first property.

The second property follows from

$$
\operatorname{Hom}_{\operatorname{Seg}_{n}}\left(F\left(U^{\prime}\right), F\left(W_{\lambda} \otimes U_{\text {spin }}\right)\right)=\operatorname{Hom}_{\mathbb{C}_{\left[\widetilde{S}_{n}\right]^{-}}}\left(U^{\prime}, G \circ F\left(W_{\lambda} \otimes U_{\text {spin }}\right)\right) \neq 0,
$$

where the last equality follows from Propositions 6.6 and 7.15.

Lemma 7.17. For a partition $\lambda$ of $n$ with distinct parts, there exists a representative $U \in \Phi_{2}(\lambda)$ such that

$$
\operatorname{Hom}_{\operatorname{Seg}_{n}}\left(U, \operatorname{Res}_{\operatorname{Seg}_{n}}^{\mathbb{H}_{n}^{\mathrm{Cl}}} X_{\lambda}\right) \neq 0
$$

Proof. This follows from

$$
\begin{aligned}
\operatorname{Hom}_{\mathrm{Seg}_{n}}\left(U, \operatorname{Res}_{\mathrm{Seg}_{n}}^{\mathbb{H}_{n}^{\mathrm{Cl}}} X_{\lambda}\right) & \\
& =\operatorname{Hom}_{\mathrm{Seg}_{n}}\left(U, F\left(\left(\mathbb{C}\left[S_{n}\right] \otimes_{\mathbb{C}\left[S_{\lambda}\right]} \text { triv }\right) \otimes U_{\text {spin }}\right)\right) \quad \text { (by Proposition 7.7) } \\
& \supseteq \operatorname{Hom}_{\mathrm{Seg}_{n}}\left(U, F\left(W_{\lambda} \otimes U_{\text {spin }}\right)\right) \quad\left(\text { by definition of } W_{\lambda}\right) .
\end{aligned}
$$

The statement now follows from Proposition 7.16. 
The following theorem states that the induced modules $\left(\pi_{\lambda}, X_{\lambda}\right)$ with $\lambda$ of distinct parts have non-zero Dirac cohomologies.

Theorem 7.18. Let $\lambda$ be a partition of $n$ with distinct parts. Let $\left(\pi_{\lambda}, X_{\lambda}\right)$ be the $\mathbb{H}_{n}^{\mathrm{Cl}}$-module defined in (7.19). Let $\Psi_{2}$ be the map defined in Proposition 7.16. Then there exists a representative $U$ in $\Psi_{2}(\lambda)$ such that

$$
\operatorname{Hom}_{\operatorname{Seg}_{n}}\left(U, H_{D}\left(X_{\lambda}\right)\right) \neq 0 \text {. }
$$

In particular, $H_{D}\left(X_{\lambda}\right)$ is non-zero.

Proof. For a fixed $\lambda \in \mathcal{P}_{n}^{\text {dist }}$, let $U$ be a $\operatorname{Seg}_{n}$-module with the property in Lemma 7.17. Then there exists a non-zero vector $v$ in the isotypical component $U$ of $X_{\lambda}$. By Theorem 4.23, Lemma 7.3, and Proposition 7.16, $\pi_{\lambda}\left(D^{2}\right) v=\left(\chi_{\pi_{\lambda}}\left(\Omega_{\mathbb{H}_{n} 1}\right)-\right.$ $\left.\chi_{\Psi_{2}(\lambda)}\left(\Omega_{\operatorname{Seg}_{n}}\right)\right) v=\left(\mathbf{k}^{2}\left|\Phi_{2}(\lambda)\right|^{2}-\chi_{\Psi_{2}(\lambda)}\left(\Omega_{\operatorname{Seg}_{n}}\right)\right) v=0$. Hence, $v \in \operatorname{ker}\left(\pi_{\lambda}\left(D^{2}\right)\right)$. By Proposition 7.13, $v \in H_{D}\left(X_{\lambda}\right)=\operatorname{ker} \pi_{\lambda}\left(D^{2}\right)$. This proves the theorem.

The Dirac cohomology $H_{D}\left(X_{\lambda}\right)$ also provides a way to realize irreducible $\operatorname{Seg}_{n^{-}}$ supermodules.

Corollary 7.19. For each of $\lambda \in \mathcal{P}_{n}^{\text {dist }}$, there exists a unique irreducible $\mathrm{Seg}_{n}$ supermodule $U$, up to the equivalence of $\sim_{\Pi}$, such that $\operatorname{Hom}_{\mathrm{Seg}_{n}}\left(U, H_{D}\left(X_{\lambda}\right)\right) \neq 0$. Let $\left[H_{D}\left(X_{\lambda}\right)\right]$ be an irreducible submodule of $H_{D}\left(X_{\lambda}\right)$. Then

$$
\operatorname{Irr}_{\text {sup }} \operatorname{Seg}_{n}=\bigsqcup_{\lambda \in \mathcal{P}_{n}^{\text {dist }}}\left\{\left[H_{D}\left(X_{\lambda}\right)\right], \Pi\left(\left[H_{D}\left(X_{\lambda}\right)\right]\right)\right\}
$$

where $\bigsqcup$ means the disjoint union.

Proof. For the first assertion, the existence has been proved in Theorem 7.18 and we only have to prove the uniqueness. Let $\left(\sigma^{\prime}, U^{\prime}\right)$ be an irreducible $\operatorname{Seg}_{n}$-module such that

$$
\operatorname{Hom}_{S_{e g}}\left(U^{\prime}, H_{D}\left(X_{\lambda}\right)\right) \neq 0 .
$$

Then $\chi_{\pi_{\lambda}}=\chi^{\sigma^{\prime}}$ by Theorem 4.25 and Theorem 7.18. On the other hand, by Proposition 7.16, $\left(\sigma^{\prime}, U^{\prime}\right)$ is in $\Phi_{2}\left(\lambda^{\prime}\right)$ for some $\lambda^{\prime} \in P_{n}^{\text {dist }}$. Then

$$
\operatorname{Hom}_{\operatorname{Seg}_{n}}\left(U^{\prime}, H_{D}\left(X_{\lambda^{\prime}}\right)\right) \neq 0
$$

and by Theorem 4.25 again, $\chi_{\pi_{\lambda^{\prime}}}=\chi^{\sigma^{\prime}}$. Thus $\chi_{\pi_{\lambda^{\prime}}}=\chi_{\pi_{\lambda}}$ and so $\lambda=\lambda^{\prime}$. This implies the uniqueness.

The second assertion follows from the first assertion and the bijectivity of $\Phi_{2}$ in Proposition 7.16.

Let $K\left(\mathbb{H}_{n}^{\mathrm{Cl}}\right)$ (resp. $\left.K\left(\mathrm{Seg}_{n}\right)\right)$ be the Grothendieck group of finite-dimensional $\mathbb{H}_{n}^{\mathrm{Cl}}$-supermodules (resp. finite-dimensional $\mathrm{Seg}_{n}$-supermodules). Then the Dirac cohomology $H_{D}$ induces a map, still denoted $H_{D}$, from $K\left(\mathbb{H}_{n}^{\mathrm{Cl}}\right)$ to $K\left(\mathrm{Seg}_{n}\right)$. Corollary 7.19 implies the following: 
Corollary 7.20. The image of $H_{D}: K\left(\mathbb{H}_{n}^{\mathrm{Cl}}\right) \rightarrow K\left(\mathrm{Seg}_{n}\right)$ has finite index in $K\left(\operatorname{Seg}_{n}\right)$.

Recall that the algebra homomorphism $\zeta: Z\left(\mathbb{H}_{n}^{\mathrm{Cl}}\right) \rightarrow Z\left(\operatorname{Seg}_{n}\right)_{0}$ is defined in Theorem 3.4. We also have:

Corollary 7.21. The map $\zeta: Z\left(\mathbb{H}_{n}^{\mathrm{Cl}}\right) \rightarrow Z\left(\mathrm{Seg}_{n}\right)_{0}$ is surjective.

Proof. It suffices to show that $\operatorname{dim}(\operatorname{im} \zeta) \geq \operatorname{dim} Z\left(\operatorname{Seg}_{n}\right)_{0}$. By Theorem 4.25 and Theorem 7.18, for any partition $\lambda \in \mathcal{P}_{n}^{\text {dist }}$, there exists $\left(\sigma_{\lambda}, U_{\lambda}\right) \in \operatorname{Irr}_{\text {sup }} \operatorname{Seg}_{n}$, such that $\chi_{\pi_{\lambda}}=\chi^{\sigma_{\lambda}}$. Since the central characters $\left\{\chi_{\pi_{\lambda}}\right\}_{\lambda \in \mathcal{P}_{n}^{\text {dist }}}$ are linearly independent over $\mathbb{C},\left\{\chi^{\sigma_{\lambda}}\right\}_{\lambda \in \mathcal{P}_{n}^{\text {dist }}}$ are also linearly independent. Then we have that $\operatorname{dim}(\operatorname{im} \zeta)$ is not less than the cardinality of $\mathcal{P}_{n}^{\text {dist }}$. Now the statement follows from the fact that $\operatorname{dim} Z\left(\operatorname{Seg}_{n}\right)_{0}$ is equal to the cardinality of $\operatorname{Irr}_{\text {sup }}\left(\operatorname{Seg}_{n}\right) / \sim_{\Pi}$, which is the same as the cardinality of $\mathcal{P}_{n}^{\text {dist }}$.

Remark 7.22. The author would like to thank Professor Weiqiang Wang for pointing out that there is a canonical surjective superalgebra morphism from $\mathbb{H}_{n}^{\mathrm{Cl}}$ to $\mathrm{Seg}_{n}[\mathrm{Kl}, \mathrm{Rem} .15 .4 .7]$. Denote the map to be $\zeta^{\prime}$. According to [Kl, Rem. 15.4.7], the map $\zeta^{\prime}$ sends $x_{i}$ to the Jucys-Murphy type element

$$
\zeta^{\prime}\left(x_{i}\right)=\sum_{1 \leq j<i} s_{i, j}\left(1-c_{i} c_{j}\right)
$$

and $\zeta^{\prime}$ is an identity on $\mathrm{Seg}_{n}$. It is straightforward to check that $\zeta^{\prime}(D)=0$. By considering

$$
z=\zeta(z)+D h+h D
$$

and applying $\zeta^{\prime}$ on both sides, $\zeta^{\prime}(z)=\zeta(z)$. Hence $\zeta^{\prime}$ agrees with $\zeta$ on $Z\left(\mathbb{H}_{n}^{\mathrm{Cl}}\right)$. The author would like to thank one of the referees for pointing out that the map $\zeta^{\prime}$ has already been proven to be surjective in $[\mathrm{Ru}]$ as a special case. This in turn gives another way to see that $\zeta$ is surjective.

\section{References}

[BCT] D. Barbasch, D. Ciubotaru, P. Trapa, Dirac cohomology for graded affine Hecke algebras, Acta. Math. 209 (2012), no. 2, 197-227.

[Be] G. Bergman, The diamond lemma for ring theory, Adv. Math. 29 (1978), 178-218.

[BK] J. Brundan, A. Kleschev, Projective representations of symmetric groups via Sergeev duality, Math. Z. 239 (2002), no. 1, 27-68.

[Ci1] D. Ciubotaru, Spin representations of Weyl groups and the Springer correspondence, J. reine angew. Math. 671 (2012), 199-222.

[Ci2] D. Ciubotaru, Dirac cohomology for symplectic reflection algebras, Selecta Math. 22 (2016), no. 1, 111-144.

[Dr] В. Г. Дринфельд, Вырожденные аффинные алгебры Гекке и янгианы, Функц. анализ и его прил. 20 (1986), вып. 1, 69-70. Engl. transl.: V. G. Drinfeld, Degenerate affine Hecke algebras and Yangians, Funct. Anal. Appl. 20 (1986), no. 1, 58-60. 
[WK] T. Khongsap, W. Wang, Hecke-Clifford algebras and spin Hecke algebras I: the classical affine type, Transform. Groups 13 (2008), 389-412.

[HP] J. S. Huang, P. Pandžić, Dirac Operators in Representation Theory, Mathematics: Theory \& Applications, Birkhäuser Boston, Boston, MA, 2006.

[HKS] D. Hill, J. Kujawa, J. Sussan, Degenerate afine Hecke-Clifford algebras and type Q Lie superalgebas, Math. Z. 268 (2011), 1091-1158.

[Kl] A. Kleshchev, Linear and Projective Representations of Symmetric Groups, Cambridge Tracts in Mathematics, Vol. 163 Cambridge University Press, Cambridge, 2005 .

[Na] M. Nazarov, Young's symmetrizers for projective representations of the symmetric group, Adv. Math. 127 (1997), no. 2, 190-257.

[Mo1] A. O. Morris, Projective representations of reflection groups. II, Proc. London Math. Soc. (3) 40 (1980), no. 3, 553-576.

[Mo2] A. O. Morris, Projective characters of exceptional Weyl groups, J. Algebra 29 (1974), 567-586.

[Re] E. W. Read, On projective representations of the finite reflections of type $\mathrm{B}_{l}$ and $\mathrm{D}_{l}$, J. London Math. Soc. (2) 10 (1975), 129-142.

[Ru] O. Ruff, Centers of cyclotomic Sergeev superalgebras, J. Algebra 331 (2011), 490511 .

[Sc] I. Schur, Uber die Darstellung der symmetrischen und der alternierenden Gruppe durch gebrochene lincare Substitutionen, J. reine andew. Math. 139 (1911), 155250 .

[Se] А. Н. Сергеев, Тензорная алгебра тождественного представления как модуль над супералгебрами Ли $\mathfrak{G} l(n, m)$ и $Q(n)$, Матем. сб. 123(165) (1984), no. 3, 422-430. Engl. transl.: A. N. Sergeev, Tensor algebra of the identity representation as a module over the Lie superalgebras $\mathfrak{G} l(n, m)$ and $Q(n)$, Math. USSR-Sbornik 51 (1985), no. 2, 419-427.

[St] J. Stembridge, Shifted tableaux and the projective representations of symmetric groups, Adv. Math. 74 (1989), 87-134.

[Wa] J. Wan, Completely splittable representations of affine Hecke-Clifford algebras, J. Algebraic Comb. 32 (2010), 15-58.

[Wan] W. Wang, Double affine Hecke algebras for the spin symmetric group, Math. Res. Lett. 16 (2009), 1071-1085.

Open Access This article is distributed under the terms of the Creative Commons Attribution 4.0 International License (http://creativecommons.org /licenses/by/4.0/), which permits unrestricted use, distribution, and reproduction in any medium, provided you give appropriate credit to the original author(s) and the source, provide a link to the Creative Commons license, and indicate if changes were made. 